This item was submitted to Loughborough's Research Repository by the author.

Items in Figshare are protected by copyright, with all rights reserved, unless otherwise indicated.

\title{
Scattering by a semi-infinite periodic array and the excitation of surface
} waves

PLEASE CITE THE PUBLISHED VERSION

LICENCE

CC BY-NC-ND 4.0

REPOSITORY RECORD

Linton, C.M., R. Porter, and lan Thompson. 2019. "Scattering by a Semi-infinite Periodic Array and the Excitation of Surface Waves”. figshare. https://hdl.handle.net/2134/2464. 


\title{
Loughborough University
}

\section{SCATTERING BY A SEMI-INFINITE PERIODIC ARRAY AND THE EXCITATION OF SURFACE WAVES}

\author{
C M LINTON*, R PORTER ${ }^{\dagger}$, AND I THOMPSON ${ }^{\ddagger}$
}

\begin{abstract}
The two-dimensional problem of acoustic scattering of an incident plane wave by a semi-infinite array of either rigid or soft circular scatterers is solved. Solutions to the corresponding infinite array problems are used, together with a novel filtering approach, to enable accurate solutions to be computed efficiently. Particular attention is focussed on the determination of the amplitude of the Rayleigh-Bloch waves that can be excited along the array. In general, the far field away from the array consists of sum of a finite number of plane waves propagating in different directions (the number depending on the observation angle) and a circular wave emanating from the edge of the array. In certain resonant cases (characterised by one of the scattered plane waves propagating parallel to the array), a different far field pattern occurs, involving contributions that are neither circular waves nor plane waves. Uniform asymptotic expansions that vary continuously across all of the shadow boundaries that exist are given for both cases.
\end{abstract}

Key words. acoustic scattering, semi-infinite array, surface wave

AMS subject classifications. 74J20, 74J15, 78A45

1. Introduction. Large array scattering problems are of considerable current interest in many different areas and present significant theoretical and computational challenges. Whereas wave scattering by a small number of scatterers, or by an infinite periodic array, is fairly well understood, scattering by large but finite arrays has received much less attention. Scattering by large finite arrays is of considerable importance in the theory of array antennas and the fabrication of electromagnetic band gap materials, [1],[2],[3]; in water waves, [4],[5] where offshore structures supported by thousands of cylindrical columns are being designed; and in acoustics, where large periodic arrays continue to be the subject of numerous studies - applications include acoustic filters, noise control, and the design of transducers.

It has long been recognised that one way to approach large finite array scattering is to analyse the effects of each edge of the array in isolation - in other words to study arrays with just one edge - and this leads to problems formulated on semi-infinite arrays. On the assumption that opposite edges of a finite array are well separated, results from analyses of semi-infinite arrays can then be combined to provide results for finite arrays. Unfortunately, such problems are difficult to analyse and little work has been done on the subject since the pioneering studies of Hills \& Karp [6] and Millar [7].

For the case of a semi-infinite periodic array of isotropic point scatterers, it has been shown [8] that progress can be made if the problem is formulated for the difference between unknowns relevant to the infinite and semi-infinite array problems. The situation considered was appropriate to acoustic diffraction by sound-soft scatterers in the limit as the ratio of wavelength to body size tends to infinity and also to the scattering of an $E$-polarized electromagnetic wave by an array of perfectly conducting wires. Important as these applications are, they do not cover many of the cases in which large array scattering is a serious issue.

As a significant extension we consider here two-dimensional scattering by a semi-

\footnotetext{
${ }^{*}$ Dept of Mathematical Sciences, Loughborough University, Leicestershire, LE11 3TU, UK

${ }^{\dagger}$ Dept of Mathematics, University of Bristol, Bristol, BS8 1TW, UK

${ }^{\ddagger}$ Dept of Mathematical Sciences, Loughborough University, Leicestershire, LE11 3TU, UK
} 
infinite row of periodically-spaced, identical circular cylinders and show how the diffracted field can be efficiently computed. We extend the techniques developed in [8] so as to investigate the effects of the size of scatterers and the boundary conditions applied on them. Neumann boundary conditions appropriate for rigid bodies give rise to a major complication since diffraction gratings of rigid structures are known to support pure Rayleigh-Bloch surface waves at low frequencies [9],[10] and these may be excited by the edge of the array.

The excitation of surface waves by array edges is a virtually unexplored area, partly because there are very few geometries for which the range of possible RayleighBloch modes (also called array guided surface waves) is completely understood. They have been observed numerically in arrays of dipoles [2] and are akin to the edge waves that can be excited by the edge of a semi-infinite crack in a thin plate [11]. It is common practice in many applications to assume that the behaviour of a large finite array can be approximated well by an infinite array, at least away from the edges. One of the consequences of the presence of Rayleigh-Bloch surface waves is that this is no longer valid. For example, Maniar and Newman [12] showed that the effect of these modes (especially those which are close to standing modes) can be extremely important, giving rise to enormous amplification of the wave field close to the centre of a large array. It is thus important to have a good understanding of when and to what extent array guided surface waves are excited.

The structure of the paper is as follows. We begin in $\S 2$ by formulating the scattering problems for both an infinite and a semi-infinite array of circular scatterers using separation of variables. Details of the Rayleigh-Bloch surface waves that can be supported by an infinite array are given. In $\S 3$ the solution to the infinite array problem is used to re-formulate the semi-infinite array problem in such a way that the unknown coefficients associated with each cylinder decay to zero as one moves away from the array edge. A number of different approaches are considered and they are used to compute the amplitude of the Rayleigh-Bloch waves that are excited. The nature of the far field is analysed in $\S 4$ and a uniform asymptotic approximation is derived. A special treatment is required when the parameters correspond to resonance in the infinite array (when one of the scattered waves propagates along the array) and this is given in $\S 5$. In particular, this allows us to solve the semi-infinite problem in the case of head-on incidence.

2. Formulation. We consider a two-dimensional scattering problem which has application in a number of physical contexts. We will refer primarily to the acoustic setting in which we look for time-harmonic solutions $\operatorname{Re}[\phi(x, y) \exp (-\mathrm{i} \omega t)]$ so that the acoustic potential $\phi$ satisfies the two-dimensional Helmholtz equation $\left(\nabla^{2}+k^{2}\right) \phi=0$ in the region exterior to the scatterers, where $k=\omega / c$ and $c$ is the speed of sound. The scatterers can be taken as either rigid (in which case the normal derivative of $\phi$ must vanish on the boundary; we call this the Neumann problem) or acoustically soft (in which case the appropriate boundary condition is $\phi=0$; we call this the Dirichlet problem). Exactly the same boundary-value problem can be used to study electromagnetic diffraction by an array of perfect conductors or, once the depth variation has been factored out, the scattering of water waves by vertical circular cylinders. In this latter case, $k$ is the positive solution to the dispersion relation $k \tanh k h=\omega^{2} / g, h$ being the water depth and $g$ the acceleration due to gravity; the appropriate boundary condition is $\partial \phi / \partial n=0$. The geometry under consideration is sketched in Figure 2.1. 

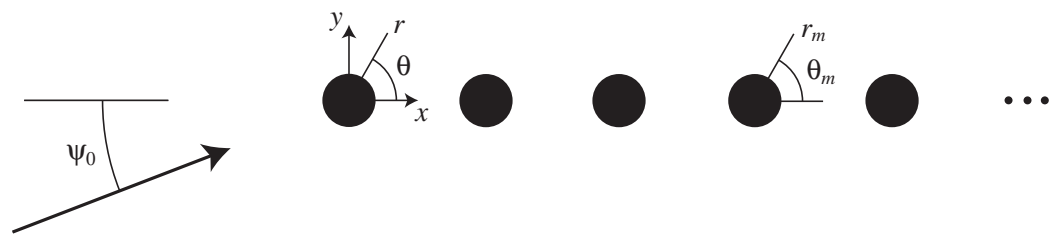

FIG. 2.1. Definition sketch

We are concerned with the scattering of a plane wave

$$
\phi_{\text {inc }}=\mathrm{e}^{\mathrm{i}(\lambda x+\mu y)} \text {, }
$$

where $\mu=k \sin \psi_{0}$ and $\lambda=k \cos \psi_{0}$, by a semi-infinite row of identical circular cylinders of radius $a$, located at $(x, y)=(j, 0), j=0,1,2, \ldots$. The spacing between the cylinders has been set to unity for convenience, and hence $0<a \leq 0.5$. We will use polar coordinates $\left(r_{j}, \theta_{j}\right)$, centred on the $j$ th scatterer and defined by

$$
x-j=r_{j} \cos \theta_{j}, \quad y=r_{j} \sin \theta_{j}
$$

and we will usually write $(r, \theta)$ for $\left(r_{0}, \theta_{0}\right)$. In terms of $\left(r_{j}, \theta_{j}\right)$ the incident wave is given by

$$
\phi_{\text {inc }}=\mathrm{e}^{\mathrm{i} \lambda j} \mathrm{e}^{\mathrm{i} k r_{j} \cos \left(\theta_{j}-\psi_{0}\right)} .
$$

This problem can be formulated using separation of variables. If we write the total field as $\phi=\phi_{\text {inc }}+\phi_{\text {sc }}$ with

$$
\phi_{\mathrm{sc}}=\sum_{j=0}^{\infty} \sum_{n=-\infty}^{\infty} A_{n}^{j} Z_{n} \mathrm{H}_{n}\left(k r_{j}\right) \mathrm{e}^{\mathrm{i} n \theta_{j}},
$$

where $\mathrm{H}_{n}(\cdot)$ is a Hankel function of the first kind and $Z_{n}=\mathrm{J}_{n}(k a) / \mathrm{H}_{n}(k a)$ if the Dirichlet problem is being studied, or $Z_{n}=\mathrm{J}_{n}^{\prime}(k a) / \mathrm{H}_{n}^{\prime}(k a)$ for the Neumann problem, then the unknowns $A_{n}^{j}$ are solutions to ([13, eqn 2.11])

$$
A_{m}^{p}+\sum_{n=-\infty}^{\infty} Z_{n} \sum_{\substack{j=0 \\ \neq p}}^{\infty} A_{n}^{j} X_{n-m}^{j p} \mathrm{H}_{n-m}(k|j-p|)=-\mathrm{e}^{\mathrm{i} \lambda p} \mathrm{e}^{\mathrm{i} m\left(\frac{1}{2} \pi-\psi_{0}\right)}
$$

$p=0,1,2, \ldots, m \in \mathbb{Z}$, where $X_{n}^{j p}=1$ if $p>j$ and $X_{n}^{j p}=(-1)^{n}$ if $p<j$.

This system of equations could, in principle, be solved numerically by truncation but the infinite spatial sum (over $j$ ) converges extremely slowly. (The coefficients $A_{n}^{j}$ do not decay to zero as $j \rightarrow \infty$ and so the terms in this sum decay like $j^{-1 / 2} \exp (\mathrm{i} j \delta)$ for some $\delta$.) By contrast, the order summation (over $n$ ) converges exponentially. The strategy that is followed here is to make use of known properties of the diffraction problem when the array extends to both plus and minus infinity to allow us to sum up the slowly convergent spatial series analytically.

For the infinite grating problem, with cylinders at $(j, 0), j \in \mathbb{Z}$, we can seek a solution of the form

$$
\phi_{\mathrm{sc}}^{\mathrm{inf}}=\sum_{j=-\infty}^{\infty} \sum_{n=-\infty}^{\infty} B_{n}^{j} Z_{n} \mathrm{H}_{n}\left(k r_{j}\right) \mathrm{e}^{\mathrm{i} n \theta_{j}}
$$


The periodicity of the geometry and of the incident wave allows us to look for a solution which satisfies

$$
B_{n}^{j}=\mathrm{e}^{\mathrm{i} \lambda j} B_{n}^{0}=\mathrm{e}^{\mathrm{i} \lambda j} B_{n},
$$

say, and then we only need to solve for $B_{n}$. These coefficients are solutions to the infinite system of equations

$$
B_{m}+\sum_{n=-\infty}^{\infty} B_{n} Z_{n} \sigma_{n-m}(\lambda)=-\mathrm{e}^{\mathrm{i} m\left(\frac{1}{2} \pi-\psi_{0}\right)}, \quad m \in \mathbb{Z},
$$

where

$$
\sigma_{n}(\lambda)=\sum_{j=1}^{\infty}\left[(-1)^{n} \mathrm{e}^{\mathrm{i} \lambda j}+\mathrm{e}^{-\mathrm{i} \lambda j}\right] \mathrm{H}_{n}(k j)
$$

The quantities $\sigma_{n}$ are easily evaluated (though not from the above expression); see $[14,15]$. The system $(2.8)$ is straightforward to solve numerically by truncation, the convergence of $B_{n}$ with $|n|$ being exponential.

The far field for the infinite array problem can be determined as follows. First we define the scattering angles $\psi_{m}(\lambda)$ by

$$
\psi_{m}=\arccos \left(\lambda_{m} / k\right), \quad \lambda_{m}=\lambda+2 m \pi .
$$

If $\left|\lambda_{m}\right|<k$, i.e.

$$
-1<\cos \psi_{0}+\frac{2 m \pi}{k}<1
$$

then we say that $m \in \mathcal{M}$ and we have $0<\psi_{m}<\pi$. If $\left|\lambda_{m}\right|>k$ then $\psi_{m}$ is no longer real and the appropriate branch of the arccos function is given by

$$
\arccos t= \begin{cases}\mathrm{i} \operatorname{arccosh} t & t>1 \\ \pi-\mathrm{i} \operatorname{arccosh}(-t) & t<-1,\end{cases}
$$

with $\operatorname{arccosh} t=\ln \left(t+\sqrt{t^{2}-1}\right)$ for $t>1$. Next we use the integral representation

$$
\mathrm{H}_{n}(k r) \mathrm{e}^{\mathrm{i} n \theta}=\frac{(-\mathrm{i})^{n+1}}{\pi} \int_{-\infty}^{\infty} \frac{\mathrm{e}^{-k \gamma(t)|y|}}{\gamma(t)} \mathrm{e}^{\mathrm{i} k x t}(t-\gamma(t))^{n \operatorname{sgn}(y)} \mathrm{d} t,
$$

in which $\gamma(t)=\left(t^{2}-1\right)^{1 / 2}$ with $\gamma(0)=-\mathrm{i}$ and the path of integration is indented so as to pass above the branch point at $t=-1$ and below that at $t=1$ (for $n=0$ see [8, Appendix A], for the extension to all $n$ we use [16, Theorem 2.7]). If this is inserted into (2.6) we can apply the Poisson summation formula to obtain

$$
\phi_{\mathrm{sc}}^{\mathrm{inf}}=\sum_{m=-\infty}^{\infty} \mathcal{F}_{m}^{ \pm} \mathrm{e}^{\mathrm{i} k r \cos \left(\theta \mp \psi_{m}\right)},
$$

in which

$$
\mathcal{F}_{m}^{ \pm}=\frac{2}{k} \sum_{n=-\infty}^{\infty}(-\mathrm{i})^{n} B_{n} Z_{n} \frac{\mathrm{e}^{ \pm \mathrm{i} n \psi_{m}}}{\sin \psi_{m}}
$$


and the superscripts + and - correspond to $y>0$ and $y<0$, respectively. The integral representation (2.13) with $n \neq 0$ is valid except on $y=0$ and so this expression for the field is valid everywhere outside the scatterers except on $y=0$, provided $\sin \psi_{m} \neq 0$ for any $m$. If there is a value of $m$ for which $\sin \psi_{m}=0$ then the scattering problem is described as resonant and requires a separate treatment. This is discussed elsewhere [17]. As $y \rightarrow \pm \infty$, the only contribution comes from those $m$ for which $\psi_{m}$ is real, i.e. $m \in \mathcal{M}$. Hence the far field consists of a set of plane waves propagating in the directions $\theta=\psi_{m}$ and $\theta=2 \pi-\psi_{m}$ :

$$
\phi_{\mathrm{sc}}^{\mathrm{inf}} \sim \sum_{m \in \mathcal{M}} \mathcal{F}_{m}^{ \pm} \mathrm{e}^{\mathrm{i} k r \cos \left(\theta \mp \psi_{m}\right)} \quad \text { as } \quad y \rightarrow \pm \infty .
$$

Crucial to what follows is the fact that in the Neumann problem the solution to the scattering problem described above may not be unique. If we relax the quasiperiodicity condition (2.7) and replace it with another with a different phase, it may possible to find a value $\beta$ (maybe more than one), dependent on $k$, such that the homogeneous infinite system

$$
B_{m}+\sum_{n=-\infty}^{\infty} B_{n} Z_{n} \sigma_{n-m}(\beta)=0, \quad m \in \mathbb{Z},
$$

has a non-trivial solution. The resulting potential

$$
\phi^{\mathrm{rb}}=\sum_{j=-\infty}^{\infty} \sum_{n=-\infty}^{\infty} \mathrm{e}^{\mathrm{i} \beta j} B_{n} Z_{n} \mathrm{H}_{n}\left(k r_{j}\right) \mathrm{e}^{\mathrm{i} n \theta_{j}}
$$

does not share the same periodicity as the incident wave, but nevertheless satisfies all the boundary conditions of the full problem. Such potentials are referred to as Rayleigh-Bloch surface waves (or array guided surface waves) and for the geometry under consideration here the dispersion relation connecting $\beta$ and $k$ has been computed in [18] and [19] (the existence of these surface waves was proved in [10]). Since $\exp (\mathrm{i} \beta m)=\exp (\mathrm{i}(\beta+2 \pi) m)$ we can restrict attention to $0 \leq \beta<2 \pi$. It follows from (2.17) that if there is a solution for a given $\beta$ then there is also a solution with $\beta$ replaced by $2 \pi-\beta$ (representing a wave whose energy is travelling in the opposite direction). If we insist that energy is propagating in the positive $x$-direction, as it will be in the semi-inifnite array problem considered below, then we can restrict attention to $0<\beta<\pi$. The numerical results in [18] and [19] show that Rayleigh-Bloch surface waves exist at discrete values of $k$ for any $\beta<\pi$ and they satisfy $k<\beta$. The fact that no such modes exist in the Dirichlet problem is proved in [20].

Computations show that a mode which is symmetric about $y=0$ (for which $\left.B_{-n}=(-1)^{n} B_{n}\right)$ exists for all scatterer sizes, and a mode antisymmetric about $y=0$ (for which $B_{-n}=-(-1)^{n} B_{n}$, with $B_{0}=0$ ) exists for $0.403 \lesssim a \leq 0.5$. For a given value of $a$, Rayleigh-Bloch waves only exist for a range of values of $k$; symmetric modes in the range $0<k<k_{\max }^{\mathrm{s}}<\pi$ and antisymmetric modes in the range $k_{\min }^{\mathrm{a}}<k<k_{\max }^{\mathrm{a}}<\pi$. It turns out that there are three distinct regimes: for $a \lesssim 0.403$ only symmetric modes are possible; for $0.403 \lesssim a \lesssim 0.459$ we have $k_{\max }^{\mathrm{s}}<k_{\min }^{\mathrm{a}}$ and so it is possible to have symmetric and antisymmetric modes, but not for the same value of $k$; finally when $0.459 \lesssim a<0.5$ we have $k_{\max }^{\mathrm{s}}>k_{\min }^{\mathrm{a}}$ and hence it is only in this parameter range that it is possible to excite both symmetric and antisymmetric modes at the same time. Figure 2.2 shows values of $k_{\max }^{\mathrm{s}}, k_{\min }^{\mathrm{a}}$ and 


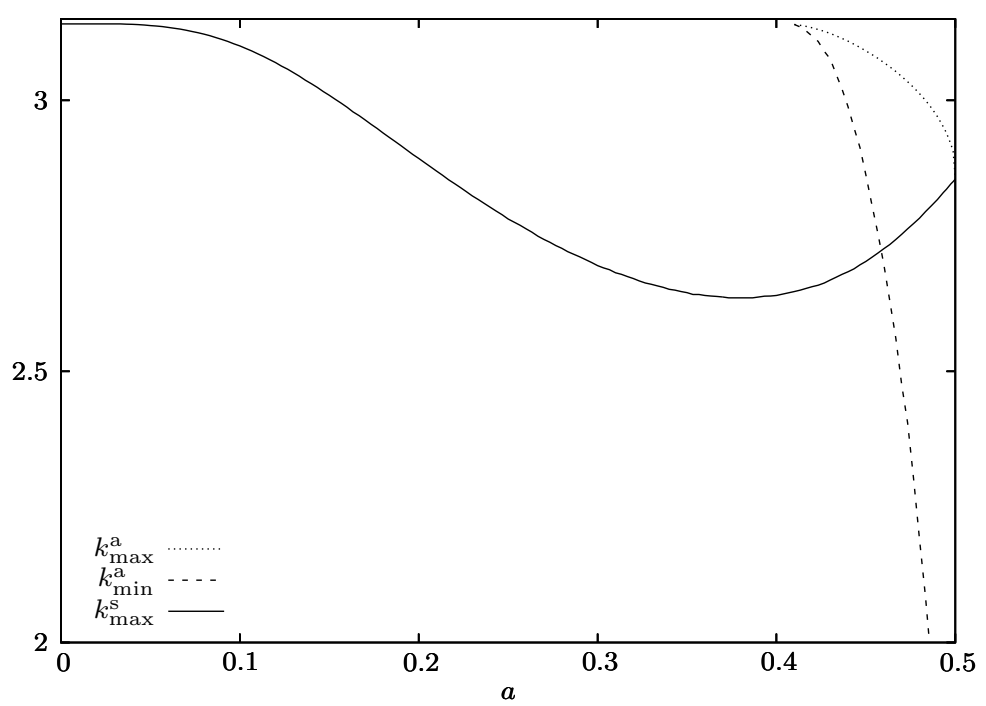

FIG. 2.2. $k_{\min }$ and $k_{\max }$ for symmetric and antisymmetric Rayleigh-Bloch modes.

$k_{\max }^{\mathrm{a}}$ for varying scatterer radius $a$. When $a=0.5$ the symmetric and antisymmetric Rayleigh-Bloch modes are essentially the same since the cylinders are touching and there is no connection between the two sides of the array.

3. Infinite array subtraction. We will formulate the problem allowing for the excitation of a single Rayleigh-Bloch mode. If both symmetric and antisymmetric modes are present, then this is easily accommodated by first splitting the problem into parts symmetric and antisymmetric about $y=0$ and treating each of them separately. In fact it is numerically efficient to make this decomposition irrespective of the parameter values and this was done in all the computations presented later. For the semi-infinite grating we would like to construct an infinite system of equations in which, unlike (2.5), the unknowns decay to zero as one moves along the array. To this end we first introduce new unknowns which are the differences between the solutions to the infinite and semi-infinite array problems (as in [8, 21]). There are then a number of different ways in which the Rayleigh-Bloch waves can be handled which fall into two broad categories. These are filtering methods, in which knowledge about the phase of the unknown coefficients is used to filter out unwanted terms (used for a simpler quasi one-dimensional scattering problem in [22]), and explicit methods, where the amplitude of the Rayleigh-Bloch waves that are excited is introduced as an extra unknown, and an extra equation is therefore required. We will consider the latter type first.

We define a new set of unknowns, $\hat{C}_{m}^{p}$, as follows:

$$
A_{m}^{p}=\hat{C}_{m}^{p}+\mathrm{e}^{\mathrm{i} \lambda p} B_{m}+\alpha \mathrm{e}^{\mathrm{i} \widetilde{\beta} p} \widetilde{B}_{m}
$$

Here $B_{m}$ is the solution to the infinite array problem $(2.8), \widetilde{B}_{m}$ is a solution to the homogeneous system (2.17) with $\beta \equiv \widetilde{\beta}$, normalised so that

$$
\sum_{m=-\infty}^{\infty}\left|Z_{m} \widetilde{B}_{m}\right|^{2}=1,
$$


and $\alpha$ is an unknown constant representing the (complex) amplitude of the RayleighBloch mode. We expect that as $p \rightarrow \infty$ (i.e. as we move away from the edge) the coefficients $A_{m}^{p}$ will tend to the values appropriate to a fully infinite array, plus possibly the effect of any Rayleigh-Bloch waves, and hence that $\hat{C}_{m}^{p} \rightarrow 0$ as $p \rightarrow \infty$ provided $\alpha$ is chosen appropriately.

If we substitute from (3.1) into (2.5) and use (2.8) and (2.17) we get a system of equations for the coefficients $\hat{C}_{m}^{p}$ which is the same as (2.5) except with a different right-hand side:

$$
\begin{aligned}
\hat{C}_{m}^{p} & +\sum_{n=-\infty}^{\infty} Z_{n} \sum_{\substack{j=0 \\
\neq p}}^{\infty} \hat{C}_{n}^{j} X_{n-m}^{j p} \mathrm{H}_{n-m}(k|j-p|) \\
& =\sum_{n=-\infty}^{\infty} Z_{n}\left(B_{n} S_{n-m}^{p}(\lambda)+\alpha \widetilde{B}_{n} S_{n-m}^{p}(\widetilde{\beta})\right), \quad p=0,1,2, \ldots, m \in \mathbb{Z}
\end{aligned}
$$

where

$$
S_{n}^{p}(\beta)=\sum_{j=p+1}^{\infty} \mathrm{e}^{\mathrm{i} \beta(p-j)} \mathrm{H}_{n}(k j) .
$$

The slowly-convergent series (3.4) does not contain unknown coefficients and so can be treated analytically, and computed efficiently; see [23]. It can be shown that (see [8], Appendix D, for the method though the final result in that paper is in error), provided $\theta$ is not an integer multiple of $2 \pi$,

$$
\sum_{j=p+1}^{\infty} \frac{\mathrm{e}^{\mathrm{i} j \theta}}{j^{1 / 2}} \sim \frac{-p^{-1 / 2} \mathrm{e}^{\mathrm{i} \theta p}}{1-\mathrm{e}^{-\mathrm{i} \theta}} \quad \text { as } \quad p \rightarrow \infty .
$$

Hence

$$
S_{n}^{p}(\beta) \sim-\sqrt{\frac{2}{\pi k p}} \frac{(-\mathrm{i})^{n} \mathrm{e}^{-\frac{1}{4} \mathrm{i} \pi} \mathrm{e}^{\mathrm{i} k p}}{\left(1-\mathrm{e}^{-\mathrm{i}(k-\beta)}\right)} .
$$

It follows that the right-hand side of (3.3) decays as $p \rightarrow \infty$, therefore the behaviour of the coefficients $\hat{C}_{n}^{p}$ in this limit must be such that the sum on the left hand side converges. In fact it turns out (see $\S 4$ ) that, as $p \rightarrow \infty$,

$$
\hat{C}_{n}^{p} \sim C_{n} p^{-3 / 2} \mathrm{e}^{\mathrm{i} k p} .
$$

The simplest way to determine $\alpha$ is to set $\hat{C}_{N}^{P}=0$, treat $\alpha$ as an unknown, and solve (3.3) for $\hat{C}_{n}^{0}, \ldots, \hat{C}_{n}^{P},|n| \leq N$, by truncation, a procedure which was used for a related problem in [24]. This works, in that as $P$ gets large the value obtained for $\alpha$ converges, but the convergence is slow, and very large values of $P$ are therefore required in order to obtain accurate results. We will refer to this approach to the determination of $\alpha$ as the direct method. We can also a subsequent result from the analysis of the far-field as a means of obtaining an additional equation. Thus, the asymptotic behaviour of the coefficients $\hat{C}_{n}^{p}$ given by (3.7) means that we must take $g(0)=0$ in (4.7) (see discussion at the end of $\S 4$ ) and so

$$
\sum_{n=-\infty}^{\infty}(-\mathrm{i})^{n} Z_{n}\left(\frac{B_{n}}{1-\mathrm{e}^{\mathrm{i}(\lambda-k)}}+\frac{\alpha \widetilde{B}_{n}}{1-\mathrm{e} \mathrm{i}(\widetilde{\beta}-k)}+\sum_{j=0}^{\infty} \hat{C}_{n}^{j} \mathrm{e}^{-\mathrm{i} k j}\right)=0 .
$$


Note that this is not an explicit formula for $\alpha$ since the coefficients $\hat{C}_{n}^{j}$ are the solutions to (3.3), which contains $\alpha$ on the right-hand side. For the antisymmetric part of the field this identity is trivially satisfied, so it is only of use for the symmetric part. The use of (3.3) combined with (3.8) will be referred to as the far-field method for the determination of $\alpha$. Unfortunately, the sum involving $\hat{C}_{n}^{j}$ converges too slowly for this approach to work well. In cases where there is no Rayleigh-Bloch wave (i.e. in the Neumann problem with $k>k_{\max }^{\mathrm{s}}$ or in the Dirichlet case), $\alpha=0$ and equation (3.8) is an identity that can be used as a check on the results. It can also be used as a numerical check in cases where Rayleigh-Bloch waves do exist, if $\alpha$ is calculated by some other means.

In order to make the best use of (3.8) we use a simple acceleration procedure. This procedure has been used wherever possible in what follows and we will refer to it as asymptotic acceleration. Thus, we substitute the asymptotic form for the coefficients $\hat{C}_{n}^{j}$ for all values of $j$ greater than the truncation parameter $j=J$, giving

$$
\sum_{j=0}^{\infty} \hat{C}_{n}^{j} \mathrm{e}^{-\mathrm{i} k j}=\sum_{j=0}^{J} \hat{C}_{n}^{j} \mathrm{e}^{-\mathrm{i} k j}+C_{n} \sum_{j=J+1}^{\infty} j^{-3 / 2}
$$

the coefficient $C_{n}$ being determined from the computed value of $\hat{C}_{n}^{J} \approx C_{n} J^{-3 / 2} \exp (\mathrm{i} k J)$ and the final sum being a generalised zeta function which can easily be computed from standard packages.

We have found that filtering methods yield the best results in terms of accuracy and efficiency and these are described next. This time we define new unknowns $C_{m}^{p}$ via

$$
A_{m}^{p}=C_{m}^{p}+\mathrm{e}^{\mathrm{i} \lambda p} B_{m}
$$

(so that $\left.C_{m}^{p}=\hat{C}_{m}^{p}+\alpha \mathrm{e}^{\mathrm{i} \widetilde{\beta} p} \widetilde{B}_{m}\right)$. Instead of (3.3) we now have

$$
C_{m}^{p}+\sum_{n=-\infty}^{\infty} Z_{n} \sum_{\substack{j=0 \\ \neq p}}^{\infty} C_{n}^{j} X_{n-m}^{j p} \mathrm{H}_{n-m}(k|j-p|)=\Gamma_{m}^{p},
$$

$p=0,1,2, \ldots, m \in \mathbb{Z}$, where for future convenience we have defined

$$
\Gamma_{m}^{p}=\sum_{n=-\infty}^{\infty} Z_{n} B_{n} S_{n-m}^{p}(\lambda)
$$

If Rayleigh-Bloch modes are excited, then the coefficients $C_{m}^{p}$ will not decay to zero as $p \rightarrow \infty$. Instead, we expect that $C_{m}^{p} \sim \mathrm{e}^{\mathrm{i} \tilde{\beta}} C_{m}^{p-1}$ in this limit. We thus introduce

$$
D_{m}^{p}= \begin{cases}C_{m}^{p}-\mathrm{e}^{\mathrm{i} \tilde{\beta}} C_{m}^{p-1}, & p=1,2, \ldots \\ C_{m}^{0} & p=0 .\end{cases}
$$

so that $D_{m}^{p}$ decays to zero as $p \rightarrow \infty$. This recurrence relation can be solved for $C_{m}^{p}$ to give

$$
C_{m}^{p}=\sum_{j=0}^{p} \mathrm{e}^{\mathrm{i} \tilde{\beta}(p-j)} D_{m}^{j}, \quad p=0,1,2, \ldots
$$


A system of equations for $D_{m}^{p}$ can then be derived in more than one way. If (3.14) is substituted into (3.11) we get

$$
\sum_{j=0}^{p} \mathrm{e}^{\mathrm{i} \tilde{\beta}(p-j)} D_{m}^{j}+\sum_{n=-\infty}^{\infty} Z_{n} \sum_{j=0}^{\infty} D_{n}^{j} \sum_{\substack{l=j \\ \neq p}}^{\infty} \mathrm{e}^{\mathrm{i} \widetilde{\beta}(l-j)} X_{n-m}^{l p} \mathrm{H}_{n-m}(k|l-p|)=\Gamma_{m}^{p},
$$

$p=0,1,2, \ldots, m \in \mathbb{Z}$. Alternatively we can combine equations in (3.11) in the obvious way so that

$$
D_{m}^{p}+\sum_{n=-\infty}^{\infty} Z_{n} \sum_{\substack{j=0 \\ \neq p}}^{\infty} D_{n}^{j} X_{n-m}^{j p} \mathrm{H}_{n-m}(k|j-p|)=\Gamma_{m}^{p}-\mathrm{e}^{\mathrm{i} \tilde{\beta}} \Gamma_{m}^{p-1},
$$

$p=1,2, \ldots, m \in \mathbb{Z}$. The system (3.16) then needs to be supplemented by an equation for $D_{m}^{0}$ which can be obtained from (3.11) with $p=0$ by substituting for $C_{n}^{j}$ from (3.14). This yields

$$
D_{m}^{0}+\sum_{n=-\infty}^{\infty}(-1)^{n-m} Z_{n} \sum_{j=0}^{\infty} D_{n}^{j} \sum_{l=\max (j, 1)}^{\infty} \mathrm{e}^{\mathrm{i} \tilde{\beta}(l-j)} \mathrm{H}_{n-m}(k l)=\Gamma_{m}^{0} .
$$

The sums over $l$ in either of the formulations can be expressed in terms of the sums $S_{n}^{p}$ defined in (3.4) and can be computed efficiently and accurately using results from [23]. The two formulations are equivalent, but we have found that the second (i.e. using (3.16) and (3.17)) is easier to implement. In either case, the finite sum in (3.14) has been interchanged with the (infinite) spatial sum in (3.11). This crucial step has the effect of continuing the filtered term (in this case the Rayleigh-Bloch mode) to infinity, so that it is unaffected by spatial truncation. This is the essence and great advantage of infinite array subtraction and filtering methods: only the part of the solution which decays as one moves along the array is subject to errors caused by spatial truncation.

Once the coefficients $D_{m}^{p}$ have been computed via truncation, the coefficients $C_{m}^{p}$ can be reconstructed from (3.14). If we truncate at $p=P$, a value for $\alpha$ can then be deduced from

$$
C_{m}^{P} \mathrm{e}^{-\mathrm{i} \widetilde{\beta} P}=\sum_{p=0}^{P} \mathrm{e}^{-\mathrm{i} \tilde{\beta} p} D_{m}^{p} \rightarrow \alpha \widetilde{B}_{m} \quad \text { as } \quad P \rightarrow \infty .
$$

The coefficients $D_{m}^{p}$ have the asymptotic behaviour $D_{m}^{p} \sim D_{m} p^{-3 / 2} \exp (\mathrm{i} k p)$ exactly as for $\hat{C}_{m}^{p}$ because they both model the behaviour of the scattered field once the Rayleigh-Bloch wave has been removed. Thus asymptotic acceleration can be used in (3.18).

It is possible to use the known asymptotic behaviour of $D_{m}^{p}$ to create a set of coefficients which decay like $p^{-5 / 2}$ by filtering again. In other words we define a new set of coefficients via

$$
E_{m}^{p}= \begin{cases}D_{m}^{p}-\mathrm{e}^{\mathrm{i} k} D_{m}^{p-1}, & p=1,2, \ldots \\ D_{m}^{0} & p=0\end{cases}
$$

Details of the resulting equations can be found in the Appendix. In most situations there is very limited gain from this second filtering. However, there are certain situations (which we will mention below) where it is absolutely essential. 
TABLE 3.1

Convergence of different methods for the determination of $|\alpha|$ for the symmetric Rayleigh-Bloch mode that is excited when $a=0.25, \psi_{0}=\pi / 10$, and $k=2$. The numbers in parentheses are the results when asymptotic acceleration is not used (note that this is not available when using the direct method). Eleven modes have been used in the order summations.

\begin{tabular}{cccccccc}
\hline \hline $\begin{array}{c}\text { spatial } \\
\text { truncation }\end{array}$ & direct & \multicolumn{2}{c}{ far-field } & \multicolumn{2}{c}{$\begin{array}{c}\text { single } \\
\text { filtering }\end{array}$} & \multicolumn{2}{c}{$\begin{array}{c}\text { double } \\
\text { filtering }\end{array}$} \\
\hline 50 & $(0.0938)$ & $(0.1148)$ & 0.1022 & $(0.0940)$ & 0.1007 & $(0.1015)$ & 0.1014 \\
100 & $(0.1015)$ & $(0.1213)$ & 0.0991 & $(0.1017)$ & 0.1017 & $(0.0999)$ & 0.1014 \\
150 & $(0.1035)$ & $(0.1022)$ & 0.1016 & $(0.1035)$ & 0.1016 & $(0.1014)$ & 0.1015 \\
200 & $(0.1020)$ & $(0.0869)$ & 0.1023 & $(0.1019)$ & 0.1015 & $(0.1018)$ & 0.1015 \\
250 & $(0.1007)$ & $(0.0972)$ & 0.1016 & $(0.1007)$ & 0.1014 & $(0.1016)$ & 0.1015 \\
300 & $(0.1010)$ & $(0.1117)$ & 0.1011 & $(0.1010)$ & 0.1015 & $(0.1014)$ & 0.1015 \\
\hline \hline
\end{tabular}

We have described four methods which can be used to determine $\alpha$; the direct method, the far-field method, single filtering, and double filtering. The final three of these can all be improved via asymptotic acceleration. Table 3.1 shows the relative performance of these different approaches for a typical, rather than an extreme, case. We have taken $a=0.25, \psi_{0}=\pi / 10$, and $k=2$. For these parameters, there is a symmetric Rayleigh-Bloch mode with $\widetilde{\beta} \approx 2.0268$. The table, which lists values of $|\alpha|$, clearly demonstrates the superiority of the filtering methods, and also the increased convergence that results from using asymptotic acceleration. An important caveat to note is that double filtering does not work well when $k$ and $\widetilde{\beta}$ are too close together. This happens for symmetric Rayleigh-Bloch waves when $k$ is small. However, this is mitigated against by the fact that in long waves smaller truncations in the order summations are necessary for a given accuracy and hence large spatial truncations can easily be used.

When antisymmetric surface waves are excited, computing $\alpha$ accurately is more of a challenge. One factor is that such modes only exist for large values of $a$ when the cylinders are close together, and this entails the use of many more terms in the order summations so as to accurately model the interactions. The second is that the problematic case $k \approx \widetilde{\beta}$ occurs not for very long waves, but when $k$ is near $k_{\min }^{\mathrm{a}}$. Table 3.2 shows the relative performance of the different approaches for computing $\alpha$ for the case $a=0.49, \psi_{0}=\pi / 10$, and $k=2.5$. For these parameters, there is an antisymmetric Rayleigh-Bloch mode with $\widetilde{\beta} \approx 2.5096$. (There is also a symmetric mode excited with $\widetilde{\beta} \approx 2.5644$ and so the problem must be decomposed into its symmetric and antisymmetric parts before the Rayleigh-Bloch amplitudes are calculated.) Note that the far-field method cannot be used in the antisymmetric case. Again, the filtering methods are seen to converge fastest as the spatial truncation is increased. It is also evident that the convergence is not as good as in the symmetric case presented in Table 3.1.

Figure 3.1 shows the variation in the amplitude of the excited symmetric surface wave with $k$, for three different angles of incidence, and with $\psi_{0}$, for three different wavenumbers, when $a=0.25$. For this value of $a$, symmetric Rayleigh-Bloch waves are excited for all $k$ in the range $0<k<k_{\max }^{\mathrm{s}} \approx 2.783$ (and antisymmetric RayleighBloch waves are never excited). For $0<k<1$ (not shown in the figure), $|\alpha|$ is essentially zero. This corresponds to wavelength-to-spacing ratios greater than $2 \pi$. As $k$ increases so the amplitude increases, reaching a maximum at $k_{\max }^{\mathrm{s}}$ (at which 
TABLE 3.2

Convergence of different methods for the determination of $|\alpha|$ for the antisymmetric RayleighBloch mode that is excited when $a=0.49, \psi_{0}=\pi / 10$, and $k=2.5$. The numbers in parentheses are the results when asymptotic acceleration is not used (note that this is not available when using the direct method). Twenty-one modes have been used in the order summations.

\begin{tabular}{cccccc}
\hline \hline $\begin{array}{c}\text { spatial } \\
\text { truncation }\end{array}$ & direct & \multicolumn{2}{c}{$\begin{array}{c}\text { single } \\
\text { filtering }\end{array}$} & \multicolumn{2}{c}{$\begin{array}{c}\text { double } \\
\text { filtering }\end{array}$} \\
\hline 50 & $(0.2212)$ & $(0.2212)$ & 0.2400 & $(0.3236)$ & 0.2597 \\
100 & $(0.2197)$ & $(0.2198)$ & 0.2380 & $(0.2571)$ & 0.2445 \\
150 & $(0.2258)$ & $(0.2259)$ & 0.2384 & $(0.2418)$ & 0.2413 \\
200 & $(0.2325)$ & $(0.2325)$ & 0.2392 & $(0.2375)$ & 0.2404 \\
250 & $(0.2379)$ & $(0.2380)$ & 0.2400 & $(0.2369)$ & 0.2402 \\
300 & $(0.2418)$ & $(0.2418)$ & 0.2405 & $(0.2377)$ & 0.2402 \\
\hline \hline
\end{tabular}
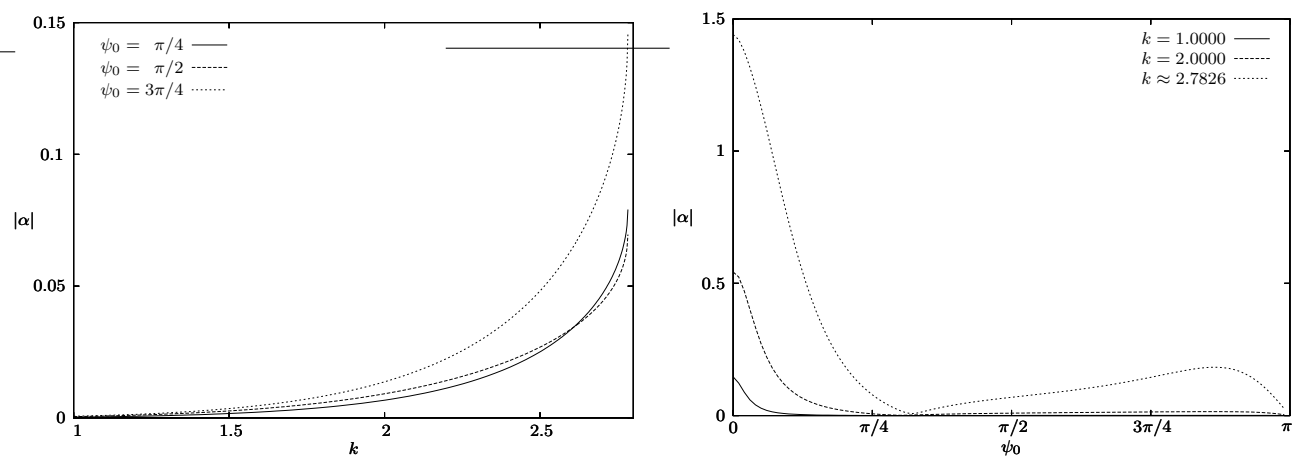

FIG. 3.1. (L) Variation with $k$ of $|\alpha|$ for the excited symmetric surface wave, for three different angles of incidence, when $a=0.25$. ( $R$ ) Variation with $\psi_{0}$ of $|\alpha|$ for the excited symmetric surface wave, for three different values of $k$, when $a=0.25$.

point $\widetilde{\beta}=\pi / 2)$. The variation in $|\alpha|$ with $\psi_{0}$ is not monotonic and this is illustrated clearly in Figure 3.1. For a given $k$, the amplitude is greatest at head-on incidence (note that this case requires special treatment as described in $\S 5$ below). It then reduces to approximately zero at an angle somewhere near $\pi / 3$ (independently of the value of $k$ ) before increasing and then getting smaller again as the incident wave grazes the array.

Figure 3.2 shows the variation in the amplitude of the excited antisymmetric surface wave with $k$, for three different angles of incidence, and with $\psi_{0}$ for three different wavenumbers, when $a=0.49$. For this value of $a$, antisymmetric RayleighBloch waves are excited for all $k$ in the range $1.796 \lesssim k \lesssim 2.969$ (and symmetric Rayleigh-Bloch waves are also excited). For $k$ just above 1.796 we have problems computing $\alpha$ accurately caused by the closeness of $k$ and $\widetilde{\beta}$. The qualitative behaviour of the amplitude as a function of $k$ is very similar to that for the symmetric mode shown in Figure 3.1. At head-on incidence the problem is entirely symmetric about the line of the array and so the amplitude of the antisymmetric mode tends to zero as the incidence angle tends to zero. The amplitude rises sharply as $\psi_{0}$ increases from zero and the maximum amplitude occurs for quite small angles.

Figures 3.1-3.2 clearly show that the amplitude of the surface waves that are excited are greatest when the frequency parameter $k$ is close to its maximum possible 

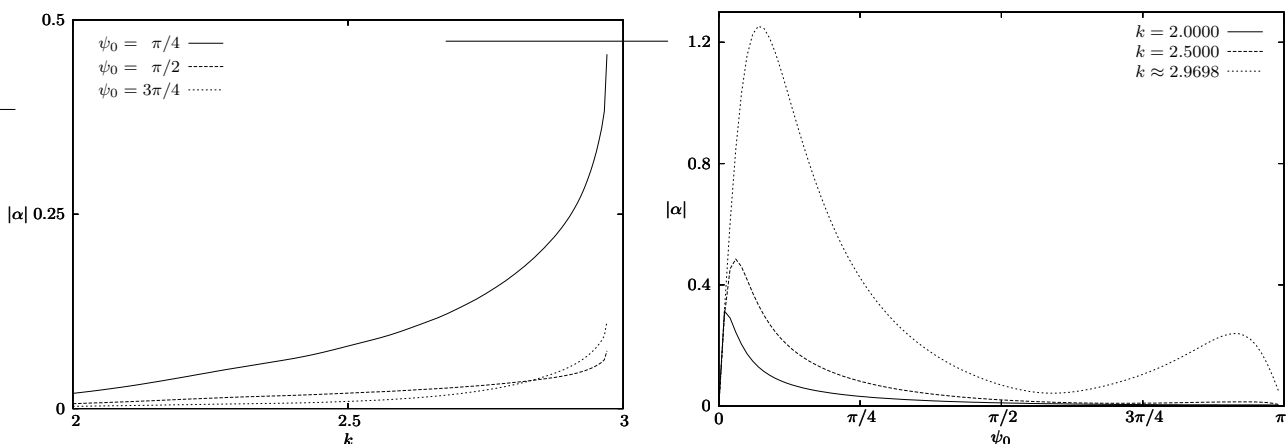

FIG. 3.2. (L) Variation with $k$ of $|\alpha|$ for the excited antisymmetric surface wave, for three different angles of incidence, when $a=0.49$. ( $R$ ) Variation with $\psi_{0}$ of $|\alpha|$ for the excited antisymmetric surface wave, for three different values of $k$, when $a=0.49$.

value for the modes to exist. When $k$ is just less than $k_{\max }, \widetilde{\beta}$ is just less than $\pi / 2$ and as $\widetilde{\beta} \rightarrow \pi / 2$ from below the Rayleigh-Bloch mode approaches a standing wave and its group velocity, $c_{\mathrm{g}}$ tends to zero. The energy in the Rayleigh-Bloch wave is proportional to $|\alpha|^{2}$ and so the rate of energy transport is proportional to $|\alpha|^{2} c_{\mathrm{g}}$ which tends to zero as $k \rightarrow k_{\max }$. Hence the large amplitudes correspond to situations where the energy is transported slowly away from the array edge.

4. The far field. From (2.4), (2.13) and (3.1) the scattered field can be written as

$$
\begin{aligned}
\phi_{\text {sc }}=\sum_{j=0}^{\infty} \sum_{n=-\infty}^{\infty}\left(\mathrm{e}^{\mathrm{i} \lambda j} B_{n}+\alpha \mathrm{e}^{\mathrm{i} \widetilde{\beta} j} \widetilde{B}_{n}+\hat{C}_{n}^{j}\right) Z_{n} \\
\times \frac{(-\mathrm{i})^{n+1}}{\pi} \int_{-\infty}^{\infty} \frac{\mathrm{e}^{-k \gamma(t)|y|}}{\gamma(t)} \mathrm{e}^{\mathrm{i} k(x-j) t}(t-\gamma(t))^{n \operatorname{sgn}(y)} \mathrm{d} t .
\end{aligned}
$$

The spatial sums involving $B_{n}$ and $\widetilde{B}_{n}$ can be evaluated using the result

$$
\sum_{j=0}^{\infty} \int_{-\infty}^{\infty} f(u) \mathrm{e}^{-\mathrm{i} j u} \mathrm{~d} u=f_{-\infty}^{\infty} \frac{f(u)}{1-\mathrm{e}^{-\mathrm{i} u}} \mathrm{~d} u
$$

(see [8], [25]), Thus

$\phi_{\text {sc }}=\sum_{n=-\infty}^{\infty} Z_{n} \frac{(-\mathrm{i})^{n+1}}{\pi} \int_{-\infty}^{\infty}\left[b_{n}(t)+\tilde{b}_{n}(t)+\hat{c}_{n}(t)\right](t-\gamma(t))^{n \operatorname{sgn}(y)} \mathrm{e}^{-k \gamma(t)|y|+\mathrm{i} k x t} \frac{\mathrm{d} t}{\gamma(t)}$,

in which

$$
\begin{aligned}
& b_{n}(t)=\frac{B_{n}}{1-\mathrm{e}^{\mathrm{i}(\lambda-k t)}}, \quad \tilde{b}_{n}(t)=\frac{\alpha \tilde{B}_{n}}{1-\mathrm{e}^{\mathrm{i}(\tilde{\beta}-k t)}} \\
& \hat{c}_{n}(t)=\sum_{j=0}^{\infty} \hat{C}_{n}^{j} \mathrm{e}^{-\mathrm{i} k j t}, \quad \operatorname{Im}(t) \leq 0 .
\end{aligned}
$$

The path of integration passes below all of the singularities, apart from the branch point at $t=-1$. 
In general the far-field asymptotics of (4.3) can be obtained via a straightforward application of the method of steepest descents, provided that the point of observation is not close to the array. The function $\hat{c}_{n}(t)$ is analytic in $\operatorname{Im}(t)<0$ by definition, and from $(3.7)$ and $[26, \S 3.4]$ the only singularities on $\mathbb{R}$ are branch points, on approach to which $\hat{c}_{n}(t)$ remains bounded; these do not contribute to the leading order far field behaviour of $\phi_{\mathrm{sc}}$. For $\operatorname{Im}(t)>0, \hat{c}_{n}(t)$ represents the meromorphic continuation of (4.5) into some cut upper half plane. While $\hat{c}_{n}(t)$ may possess singularities in this region, these will yield an exponentially small contribution to the far field should they be encountered in the process of making the steepest descents deformation. Also, the poles of $\tilde{b}_{n}(t)$ lie outside the interval $[-1,1]$, so that their contribution is also exponentially small. Thus, only the term involving $b_{n}(t)$ requires special treatment. First, assume that the saddle point $t=\cos \theta$ does not coincide with any of the poles of $b_{n}(t)$. Ignoring evanescent contributions, we find that as $k r \rightarrow \infty$ with $\theta \in(0,2 \pi)$ (provided $\psi_{m} \neq 0$ for any $m$ )

$$
\phi_{\mathrm{sc}} \sim \widetilde{H}(k r) g(\theta)+\sum_{\substack{m \in \mathcal{M} \\ \psi_{m}>\theta}} \mathcal{F}_{m}^{+} \mathrm{e}^{\mathrm{i} k r \cos \left(\theta-\psi_{m}\right)}+\sum_{\substack{m \in \mathcal{M} \\ 2 \pi-\psi_{m}<\theta}} \mathcal{F}_{m}^{-} \mathrm{e}^{\mathrm{i} k r \cos \left(\theta+\psi_{m}\right)} .
$$

Here, $\mathcal{F}_{m}^{ \pm}$is defined in $(2.15), \widetilde{H}(k r)=\sqrt{2 / \pi k r} \exp \left(\mathrm{i}\left(k r-\frac{1}{4} \pi\right)\right)$ and

$$
g(\theta)=\sum_{n=-\infty}^{\infty}(-\mathrm{i})^{n} \mathrm{e}^{\mathrm{i} n \theta} Z_{n}\left(b_{n}(\cos \theta)+\tilde{b}_{n}(\cos \theta)+\hat{c}_{n}(\cos \theta)\right) .
$$

The diffracted field takes the form of a circular wave of directivity $g(\theta)$ plus a sum of plane waves which propagate in the same directions as for the infinite grating case. However, unlike in the grating problem, the plane waves do not exist everywhere and the wave making an angle $\psi_{m}$ (resp. $\left.-\psi_{m}\right)$ with the $x$-axis is only found in the sector $0<\theta<\psi_{m}$ (resp. $2 \pi>\theta>2 \pi-\psi_{m}$ ). Crucially, the coefficients $\hat{C}_{n}^{m}$ affect only the circular wave. The plane-wave field is determined entirely from the solution to the infinite grating problem; in fact, where the plane waves exist, their amplitude is precisely as in the infinite grating problem. Thus it is only the circular wave which causes any computational difficulties.

The approximation (4.6) is non-uniform in the sense that $b_{n}(\cos \theta)$ is singular at the shadow boundaries where $\theta=\psi_{p}$ or $\theta=2 \pi-\psi_{p}$ (a case which corresponds to a pole of $b_{n}(t)$ coinciding with the saddle point). This limitation can be overcome by adding correction terms, each of which includes an error function which rapidly but continuously activates and deactivates the appropriate plane wave as the shadow boundary is crossed. These correction terms have appeared in the literature in numerous forms, and with various regions of validity. The appropriate form for use here is that given by Thompson [27], since this accounts for limits in which a shadow boundary in $y>0$ approaches its counterpart in $y<0$. Thus, the uniform approximation to $\phi_{\mathrm{sc}}$ is

$$
\begin{aligned}
\phi_{\text {sc }} \sim \widetilde{H}(k r) g(\theta)+\frac{1}{2} \mathrm{e}^{\mathrm{i} k r} \sum_{m \in \mathcal{M}}\left[\mathcal{F}_{m}^{+}\left(w\left(\zeta_{m}^{-} \mathrm{e}^{\mathrm{i} \pi / 4}\right)-\frac{\mathrm{e}^{\mathrm{i} \pi / 4}}{\zeta_{m}^{-} \sqrt{\pi}}\right)\right. & \\
& \left.+\mathcal{F}_{m}^{-}\left(w\left(\zeta_{m}^{+} \mathrm{e}^{\mathrm{i} \pi / 4}\right)-\frac{\mathrm{e}^{\mathrm{i} \pi / 4}}{\zeta_{m}^{+} \sqrt{\pi}}\right)\right],
\end{aligned}
$$

where $\zeta_{m}^{ \pm}=\sqrt{2 k r} \sin \frac{1}{2}\left(\theta \pm \psi_{m}\right)$ and $w(z)=\exp \left(-z^{2}\right) \operatorname{erfc}(-\mathrm{i} z)$ is the scaled complex error function. It is not difficult to show that (4.8) is continuous at all of the shadow 
TABLE 4.1

Propagating mode amplitudes and directions for the contour plot shown in Figure 4.1.

\begin{tabular}{r|ccc}
$j$ & $\psi_{j}$ & $\left|\mathcal{F}_{j}^{+}\right|$ & $\left|\mathcal{F}_{j}^{-}\right|$ \\
\hline-1 & $0.69 \pi$ & 0.177 & 0.578 \\
0 & $0.25 \pi$ & 0.170 & 0.734
\end{tabular}

boundaries, as the singularities in $\widetilde{H}(k r) g(\theta)$ are cancelled by those in the series (note that $w(0)=1)$. Now, if $\zeta_{m}^{ \pm}>0$, we can use the result [28, 7.1.23]

$$
w(z) \sim \mathrm{i} /(z \sqrt{\pi})+O\left(z^{-3 / 2}\right), \quad z \rightarrow \infty, \quad-\pi / 4<\arg (z)<5 \pi / 4,
$$

to show that the correction term vanishes to leading order as $k r \rightarrow \infty$. On the other hand, for $\zeta_{p}<0$, we must first apply the identity

$$
w(z)+w(-z)=2 \mathrm{e}^{-z^{2}}
$$

and then use (4.9) to deduce that

$$
\frac{\mathrm{e}^{\mathrm{i} k r}}{2}\left(w\left(\zeta_{p}^{ \pm} \mathrm{e}^{\mathrm{i} \pi / 4}\right)-\frac{\mathrm{e}^{\mathrm{i} \pi / 4}}{\zeta_{p}^{ \pm} \sqrt{\pi}}\right) \sim \mathrm{e}^{\mathrm{i} k r \cos \left(\theta \pm \psi_{p}\right)}+O\left((k r)^{-3 / 2}\right)
$$

as $k r \rightarrow \infty$. Thus each error function term in (4.8) (which is an exact solution to the Helmholtz equation) includes a plane wave in the appropriate region.

Note that the limit $y \rightarrow 0$ of (4.3) can be taken directly, provided that $x<0$, since then convergence can be maintained by deforming the path of integration into the lower half plane. The value taken for $\operatorname{sgn}(0)$ is immaterial. To see this, subtract (4.3) with $y=0^{+}$from the same equation with $y=0^{-}$. The resulting integrand has no branch point at $t=-1$, in view of the identity $(t-\gamma(t))^{n}=(t+\gamma(t))^{-n}$, and therefore evaluates to zero. This shows that (4.6) and (4.8) are valid at $\theta=\pi$. For $x>0$, the required upwards deformation cannot be performed since the sums of residues from $b(t)$ and $\tilde{b}(t)$, and of branch point contributions from $\hat{c}_{n}(t)$ all diverge when $y=0$. Consequently, (4.8) represents only a part of the far field at $\theta=0$ and $\theta=2 \pi$, and not necessarily the most significant.

Figure 4.1 shows a contour plot of the real part of the scattered field, with $a=$ $0.25, \psi_{0}=0.25 \pi$, and $k=5.0$, using Dirichlet boundary conditions. Table 4.1 contains the amplitudes of the propagating modes for this case and the real part of the leading order contribution to the far field given by (4.8) is plotted in Figure 4.2, with $r=5$. The imaginary part exhibits qualitatively similar behaviour, and is not shown. The black disks indicate the locations of the shadow boundaries. The data from which the dashed line is plotted includes contributions from plane waves, and is therefore continuous. The solid line represents the same uniform approximation, but with the plane waves removed via (4.10). The two lines coincide in the region $0.69 \pi<\theta<1.25 \pi$ where no plane waves exist; here the circular wave is clearly visible in Figure 4.1. The size of the discontinuities in the solid line is consistent with the mode amplitudes in Table 4.1; all of the associated shadow boundaries are clearly evident in Figure 4.1.

Finally, we give some justification for the asymptotic behaviour of $\hat{C}_{n}^{p}$ as $p \rightarrow \infty$ given in equation (3.7), though this will not amount to a rigourous proof. The total field in the region $r_{p}<1 / 2$ (i.e. local to scatterer $p$ ) can be written in the form [13]

$$
\phi=\sum_{n=-\infty}^{\infty} A_{n}^{p}\left[Z_{n} \mathrm{H}_{n}\left(k r_{p}\right)-\mathrm{J}_{n}\left(k r_{p}\right)\right] \mathrm{e}^{\mathrm{i} n \theta_{p}} .
$$




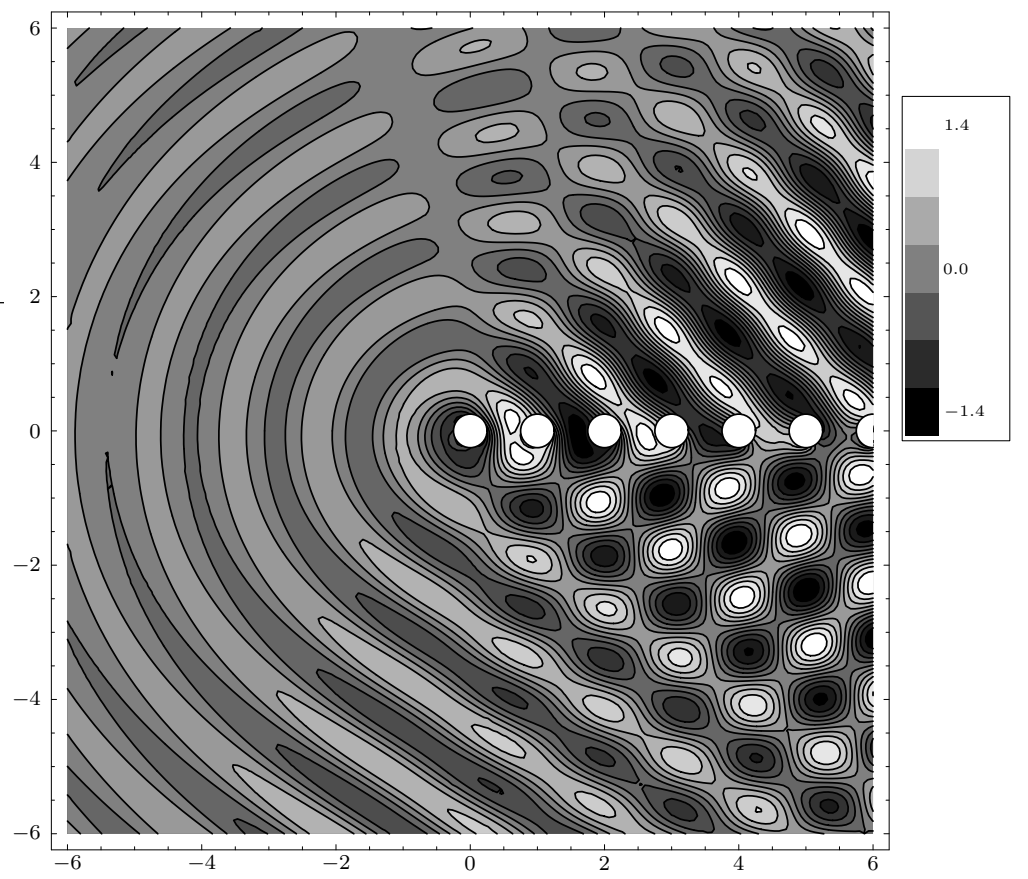

FIG. 4.1. Contour plot of $\operatorname{Re}\left[\phi_{\mathrm{sc}}\right]$, with $a=0.25, \psi_{0}=0.25 \pi k=5.0$, using Dirichlet boundary conditions.

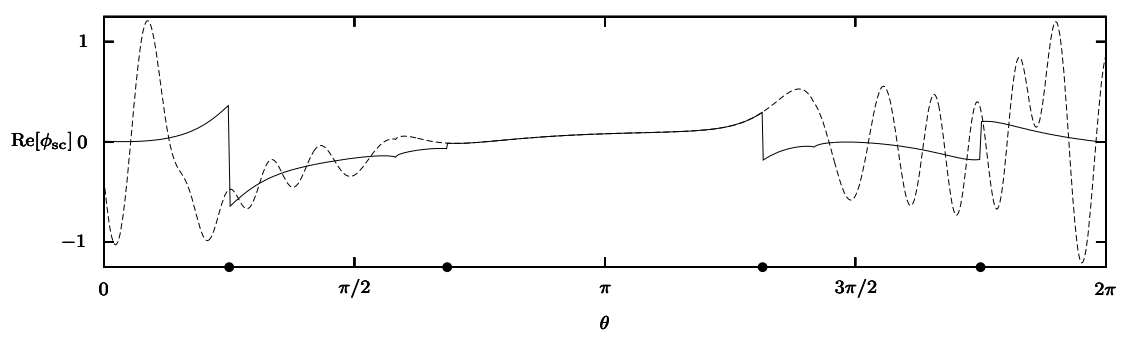

Fig. 4.2. Far field plots for the parameters used in Figure 4.1. Correction terms are included for all shadow boundaries; the dashed line includes plane wave contributions, whereas the solid line does not.

If we write similar expressions for $\phi^{\text {inf }}$ and $\phi^{\text {rb }}$ (see $\left.\S 2\right)$, and introduce $\hat{\phi}=\phi-\left(\phi^{\text {inf }}+\right.$ $\left.\phi^{\mathrm{rb}}\right)$, then from (3.1) we have

$$
\hat{\phi}=\sum_{n=-\infty}^{\infty} \hat{C}_{n}^{p}\left[Z_{n} \mathrm{H}_{n}\left(k r_{p}\right)-\mathrm{J}_{n}\left(k r_{p}\right)\right] \mathrm{e}^{\mathrm{i} n \theta_{p}} .
$$

For sufficiently large $p, \hat{\phi}$ represents the field due to end effects other than the Rayleigh-Bloch wave, and its asymptotic behaviour is evidently determined by that of $\hat{C}_{n}^{p}$. It is not difficult to show that $\hat{\phi}$ can also be represented by (4.3), but with the path of integration now passing above the poles. Nevertheless, we cannot set $y=0$, since then the sum of contributions from the branch points of $\hat{c}_{n}(t)$ would diverge. Instead, we can impose the restriction $y<a$, so that letting $x \rightarrow \infty$ causes $\theta$ to approach zero. Then, we deform the contour into the upper half plane and deduce 
the leading order behaviour of $\hat{\phi}$ as $x \rightarrow \infty$. The phase dependence and rate of decay of $\hat{C}_{n}^{p}$ as $p \rightarrow \infty$ must be such that the result of this calculation is consistent with (4.13). Now, introduce the ansatz

$$
\hat{C}_{n}^{p} \sim C_{n} \mathrm{e}^{\mathrm{i} p \xi} p^{-u}
$$

in which $C_{n}$ is a constant, $u>0$ and $\xi>0$, and substitute this into (4.5). Clearly, the critical points will be those at which the phase disappears, therefore we define

$$
t_{m}=(\xi+2 m \pi) / k
$$

The behaviour of $\hat{c}_{n}(t)$ in the vicinity of $t=t_{m}$ now follows from [26, §3.4]. Thus, as $\left(t_{m}-t\right) \rightarrow 0^{+}$, we have

$$
\hat{c}_{n}(t)=C_{n} \Gamma(1-u) \mathrm{e}^{\mathrm{i} \pi(1-u) / 2} \xi^{u-1}\left(t_{m}-t\right)^{u-1}+f_{n}(t), \quad u \neq 1
$$

where $f_{n}(t)$ is regular at $t=t_{m}$, and the terms with exponent $u-1$ are positive real. If $u=1$ then the integrand in (4.3) possesses logarithmic singularities whose contribution cannot be consistent with (4.13). There are now two cases to consider. If $\xi \neq k$, then the leading order behaviour must be due to a singularity of the function $\hat{c}_{n}(t)$, since the contribution from the branch point at $t=1$ (which in general is $\left.O\left((k r)^{-1 / 2}\right)\right)$ has the wrong phase, according to (4.13). Consequently, we must have $u<1 / 2$ in this case. On the other hand, if $\xi=k$, then the singularity at $t=1$ yields a contribution whose rate of decay is slower than that predicted by (4.13), unless a sufficient number of terms vanish as $\theta \rightarrow 0$ (or $2 \pi$ ) so as to achieve consistency. Note that we must have $u>1 / 2$, or else the the sum from $j=0$ to $j=p-1$ on the left-hand side of (3.3) would diverge as $p \rightarrow \infty$ due to phase cancellation. If $u=3 / 2$, only the leading order term must disappear, that is $g(0)=g(2 \pi)=0$, and now both (4.3) and (4.13) predict that the leading order far-field behaviour of $\hat{\phi}$ on the array is $O\left((k r)^{-3 / 2}\right)$. This is borne out by numerical computations and is also the behaviour proved in [8] using the discrete Wiener-Hopf technique for a semi-infinite array of point scatterers (which is equivalent to taking only the monopole terms in the Dirichlet problem here).

5. Resonance. Resonance occurs when one of the modes in (4.6) propagates in a direction parallel to the array. This requires that either $\psi_{m}=0$, or $\psi_{m}=\pi$ for some $m$; see (2.10). In general resonances can only occur if $k>\pi$, thereby precluding the possibility of simultaneous occurrence with Rayleigh-Bloch waves. The special case of head-on incidence $\left(\psi_{0}=0\right)$ is resonant for all $k$; symmetric Rayleigh-Bloch waves may be excited if $k<\pi$. We will take (3.11) as the starting point for solving resonant problems. Now, in order to use the infinite array subtraction technique we must first compute the coefficients $B_{n}$ in the resonant case, this is complicated by the fact that the Schlömilch series in (2.9) are now divergent. However, it can be achieved using the method developed in [17]. The form for the mode amplitude $\mathcal{F}_{m}^{ \pm}$ in the limit $\sin \psi_{m} \rightarrow 0$ is also given in [17]. It was noted in [6, 29] that for point scatterers all the nonresonant scattered modes disappear at resonance. To see this, one need only note that the solution for point scatterers is retrieved by truncating all order summations at zero. Then, at a resonance, the divergence of the Schlomilch series in (2.8) requires that $B_{0}=0$, which in turn implies that $\mathcal{F}_{p}^{ \pm}=0$, unless mode $p$ is resonant in which case we have $\mathcal{F}_{p}^{ \pm}=-1$; see [17]. For finite size scatterers, however, singular behaviour in the Schlömilch series requires that the coefficients $B_{n}$ 
TABLE 5.1

Propagating mode directions and amplitudes for the contour plot shown in Figure 5.1.

\begin{tabular}{r|ccc}
$j$ & $\psi_{j}$ & $\left|\mathcal{F}_{j}^{+}\right|$ & $\left|\mathcal{F}_{j}^{-}\right|$ \\
\hline-1 & $\pi$ & 1.46 & 0.680 \\
0 & $0.6 \pi$ & 0.620 & 0.589 \\
1 & $0.38 \pi$ & 0.180 & 0.336
\end{tabular}

satisfy a modified system of equations which permits nonzero values (except in the case of head-on incidence) and so all the modes are present in the scattered field.

The case in which $\psi_{m}=\pi$ is known as outward resonance, since now mode $m$ has the form $\mathcal{F}_{m}^{ \pm} \mathrm{e}^{-\mathrm{i} k x}$. This can only occur if $k>\pi$, therefore no Rayleigh-Bloch waves are excited. Outward resonant modes exist in all space, however in general they have different amplitudes in regions $y<0$ and $y>0$. Once the resonant solution to the infinite array problem has been obtained, no further special treatment is required, and the coefficients $C_{m}^{p}$ can be computed from (3.11). Note that, in the case of point scatterers, in which $B_{0}=0$, the right-hand side of (3.11) vanishes, so that $C_{0}^{p}=0$ for all $p$, and therefore the circular wave term in (4.6) also disappears. For finite sized scatterers, this is not the case, since $B_{n} \neq 0$ in general.

Figure 5.1 shows a contour plot of the real part of the scattered field, with $a=$ $0.25, \psi_{0}=0.6 \pi$ and the wavenumber chosen so that mode -1 is outwards resonant $(k \approx 9.1)$. Including the resonant mode, there are three propagating plane waves; the amplitudes above and below the array are shown in Table 5.1. Since the amplitude of mode 1 is relatively small, its shadow boundaries are not visible in Figure 5.1, however those at $\theta=0.6 \pi, \theta=\pi$ and $\theta=1.4 \pi$ are clearly evident. The presence of mode 0 when $\theta<0.6 \pi$ and $\theta>1.4 \pi$ accounts for the interference in this region. The real part of the leading order contribution to the far field given by (4.8) is plotted in Figure 5.2, with $r=5$. As before, the black disks indicate the locations of the shadow boundaries, the dashed line includes contributions from plane waves, whereas the solid line does not. Notice in particular the smooth transition that occurs in the amplitude of the resonant mode across $\theta=\pi$. This effect is due to the coincidence of two shadow boundaries directly opposite the array; thus, as the observer moves from the region where $\theta<\pi$ to that where $\theta>\pi$, the mode $\mathcal{F}^{+} \mathrm{e}^{-\mathrm{i} k x}$ is deactivated, and $\mathcal{F}^{-} \mathrm{e}^{-\mathrm{i} k x}$ is activated in its place. The size of the discontinuities that occur at the shadow boundaries is consistent with the mode amplitudes shown in Table 5.1. Note that the plane wave terms are, in general, of greater amplitude than the circular wave; this is why the latter is not particularly visible in Figure 5.1.

The inward resonance case in which $\psi_{m}=0$ is more interesting and presents more of a challenge. The extra difficulty in handling inward resonance was noted by Hills [29], who attempted to analyse this case for a semi-infinite array of isotropic point scatterers. As before, we require the coefficients $B_{n}$ from the infinite array problem, and these can be obtained using the method in [17]. There is now an additional obstacle, caused by the divergence of the series in the right hand side of (3.11). In fact, it can be deduced from equations in [23] that

$$
S_{n}^{p}(\lambda)=\hat{S}_{n}^{p}(\lambda)+2(-\mathrm{i})^{n} \mathrm{e}^{\mathrm{i} p k} /\left(k \psi_{m}\right),
$$

where $\hat{S}_{n}^{p}(\lambda)$ remains bounded as $\psi_{m} \rightarrow 0$, and we have used the fact that, since mode $m$ is resonant, $\cos \psi_{0}=(1-2 m \pi) / k$. In order for the solution to remain bounded, 


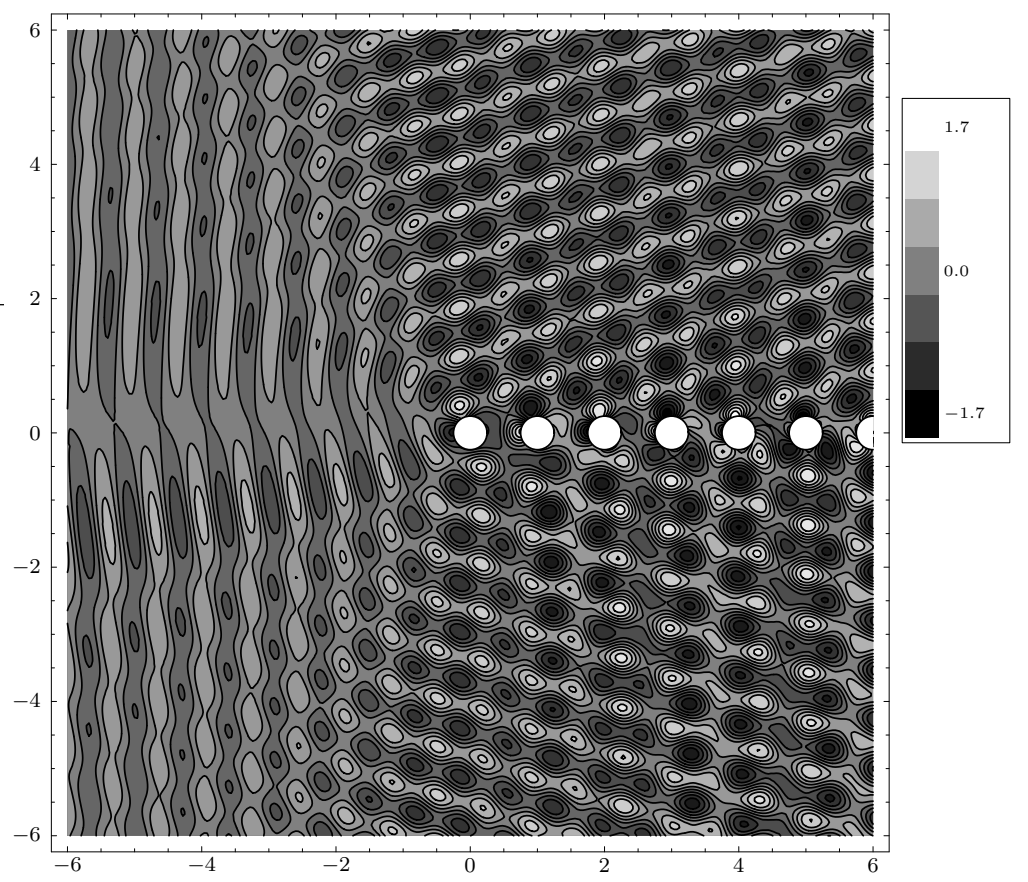

FIG. 5.1. Contour plot of $\operatorname{Re}\left[\phi_{\mathrm{sc}}\right]$, with $a=0.25, \psi_{0}=0.6 \pi$ and the wavenumber chosen so that mode -1 is (outwards) resonant $(k \approx 9.1)$.

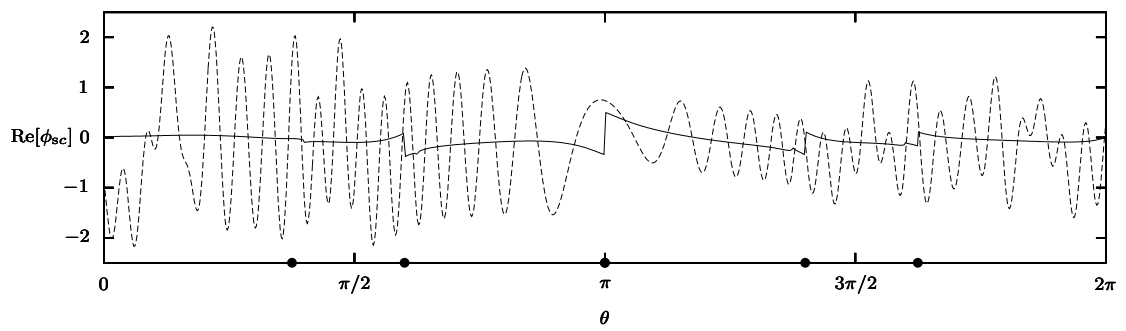

FIG. 5.2. Far field plots for the parameters used in Figure 5.1, with $r=5$. Correction terms are included for all shadow boundaries; the dashed line includes plane wave contributions, whereas the solid line does not.

we must have

$$
\frac{2}{k} \sum_{n=-\infty}^{\infty}(-\mathrm{i})^{n} Z_{n} B_{n}=a_{1} \psi_{m}+O\left(\psi_{m}^{2}\right)
$$

and the value of the constant $a_{1}$ is obtained as a biproduct of the procedure used in computing $B_{n}$; see [17]. Equation (3.11) now becomes

$$
C_{m}^{p}+\sum_{n=-\infty}^{\infty} Z_{n} \sum_{\substack{j=0 \\ \neq p}}^{\infty} C_{n}^{j} X_{n-m}^{j p} \mathrm{H}_{n-m}(k|j-p|)=a_{1} \mathrm{i}^{m} \mathrm{e}^{\mathrm{i} p k}+\sum_{n=-\infty}^{\infty} Z_{n} B_{n} \hat{S}_{n-m}^{p}(\lambda) .
$$

The solution to this linear system for an inward resonant case is composed from a sum of two components, one corresponding to each term on the right hand side of (5.3). 
The first component is a constant multiple of the solution to the head-on incidence problem, therefore the case where $\psi_{0}=0$ is canonical to all inward resonances at the same frequency. In particular, this means that the most interesting features of the scattered field at inward resonance are purely symmetric, and this is a significant simplification, as we shall see. The second component is akin to the solution of an ordinary (nonresonant) scattering problem; its computation presents no special difficulty beyond those already discussed.

We now consider the head-on incidence case in detail. Note that subtraction of the infinite array solution is not required here, since $B_{n}=0$ for all $n$ [17]. Indeed, we also have $a_{1}=-1$, therefore (5.3) is identical to (2.5). Also, the integrand in (4.3) no longer has poles at the points $t=\cos \psi_{m}, m \neq 0$, and therefore the scattered plane waves disappear in this case.

It turns out that the coefficients $\hat{C}_{n}^{p}$ decay more slowly as $p$ increases than in the non-resonant cases discussed above. To see this, we must consider the boundary condition on the surface of the scatterers for large $p$. Therefore, we may leave aside for the present the possibility of Rayleigh-Bloch waves, since these independently satisfy the boundary conditions in the far field. Next, impose the restriction $y<a$, and take the limit $x \rightarrow \infty$. Since the incident wave $\mathrm{e}^{\mathrm{i} k x}$ is present everywhere, it follows that there must be a simple pole above the path of integration in (4.3); otherwise $\phi_{\mathrm{sc}} \rightarrow 0$ and the boundary condition cannot be satisfied. In order for its contribution to possess the correct $x$ dependence, this singularity must be located at $t=1$. Indeed, from (4.14-4.16), we must have

$$
\hat{C}_{n}^{p}=C_{n}^{p} \sim C_{n} p^{-1 / 2} \mathrm{e}^{\mathrm{i} k p}
$$

as $p \rightarrow \infty$, so that $\hat{c}_{n}(t)$ has a branch point at $t=1$, and the ratio $\hat{c}_{n}(t) / \gamma(t)$ has the required simple pole. The residue can also be deduced, thus from (4.3), (4.16) and (5.4),

$$
\sum_{n=-\infty}^{\infty}(-\mathrm{i})^{n} Z_{n} C_{n}=-\sqrt{k /(2 \pi)} \mathrm{e}^{\mathrm{i} \pi / 4}
$$

so that

$$
\phi \sim \mathrm{e}^{\mathrm{i} k x}-\mathrm{e}^{\mathrm{i} k r \cos \theta} \rightarrow 0
$$

as $x \rightarrow \infty$. If the frequency is sufficiently low to permit the excitation of RayleighBloch waves, double filtering can be applied to (5.3); only the right-hand side differs from (3.11). Indeed, of the methods discussed in section 3, only this yields accurate results in this case. Results in the appendix show that the coefficients $C_{n}$ can be approximated via

$$
C_{n}=\lim _{p \rightarrow \infty} \mathrm{e}^{-\mathrm{i} k p} C_{m}^{p} ;
$$

the computed values can then be checked using (5.5). At higher frequencies, i.e. for $k>\pi$, single filtering can be used with $k$ in place of $\tilde{\beta}$. The values for $C_{n}$ can then be approximated using the limit

$$
C_{n}=\lim _{p \rightarrow \infty} \sqrt{p} \sum_{j=0}^{p} \mathrm{e}^{-\mathrm{i} k j} D_{m}^{j} .
$$


Since $\hat{c}_{n}(t)$ is $2 \pi / k$ periodic, it is evident that there are now branch points located at

$$
t=\cos \psi_{m}=1+2 m \pi / k, \quad m \neq 0
$$

The path of integration in (4.3) is indented so as to pass below these singularities. We can deduce from (4.16) and (5.4) that

$$
\hat{c}_{n}(t)=\frac{C_{n} \mathrm{e}^{-\mathrm{i} \pi / 4} \sqrt{\pi / k}}{\left(t-\cos \psi_{m}\right)^{1 / 2}}+f_{n}(t),
$$

where $f_{n}(t)$ is regular in the vicinity of the point $t=\cos \psi_{m}$, and the branch of the fractional power is chosen so that $\left(t-\cos \psi_{m}\right)^{1 / 2}=\sqrt{t-\cos \psi_{m}}$ for $t>\cos \psi_{m}$. Now the asymptotic behaviour of an integral with branch points (that are not branch points of the exponent) is far more complicated than that of an integral with poles. The essential reason for this is that a rational function can easily be split into partial fractions, whereas a product of square roots cannot. Therefore we make the assumption that the neighbourhood of the point $t=\cos \psi_{m}$ in which $f_{n}(t)$ is analytic is of sufficient size to permit the branch points of $\hat{c}_{n}(t)$ to be treated separately. This is valid if $k$ is not too large. Of course, if $k<\pi$, the branch points of $\hat{c}_{n}(t)$ given by (5.9) lie outside the interval $[-1,1]$, and are of no concern since their contribution to the far field is evanescent. Otherwise, if $\cos \psi_{m} \in(-1,1)$ then we shall write $m \in \mathcal{M}$, as before. Note that this requires $m<0$, and $k>-m \pi$.

When the saddle point in (4.3) lies to the right of a branch point (other than $t=-1$ ) then the steepest descent path is diverted in an anticlockwise loop around the cut, and an extra contribution must be included in the far field. The contribution from $t=\cos \psi_{m}$ is therefore present only in the regions where $\theta<\psi_{m}$ and $\theta>2 \pi-\psi_{m}$, which is the behaviour exhibited by the scattered plane waves in the nonresonant case. Since the field is symmetric at head on incidence, we give results for $y \geq 0$ only. Provided that we are not close to the array, we have, from (4.3) and (5.10),

$$
\phi_{\mathrm{sc}} \sim \sqrt{\frac{2}{\pi k r}} \mathrm{e}^{-\mathrm{i} \pi / 4}\left[h(\theta) \mathrm{e}^{\mathrm{i} k r}+\sum_{\substack{m \in \mathcal{M} \\ \psi_{m}>\theta}} \frac{\mathcal{G}\left(\psi_{m}\right) \mathrm{e}^{\mathrm{i} k r \cos \left(\theta-\psi_{m}\right)}}{\sqrt{\sin \left(\psi_{m}-\theta\right)}}\right],
$$

in which

$$
h(\theta)=\sum_{n=-\infty}^{\infty}(-\mathrm{i})^{n} Z_{n} \hat{c}_{n}(\cos \theta) \mathrm{e}^{\mathrm{i} n \theta},
$$

and

$$
\mathcal{G}(\psi)=\sqrt{\frac{2 \pi}{k \sin \psi}} \mathrm{e}^{\mathrm{i} \pi / 4} \sum_{n=-\infty}^{\infty} Z_{n} C_{n}(-\mathrm{i})^{n} \mathrm{e}^{\mathrm{i} n \psi}
$$

Thus, the branch point contribution is not a circular wave, as its crests are linear, perpendicular to the line $\theta=\psi_{m}$. Note from equation (5.9) that $\mathrm{e}^{\mathrm{i} n \psi_{m}}=1+O\left(k^{-1}\right)$, it then follows from (5.5) that a multiplicative factor $\mathcal{G}\left(\psi_{m}\right)$ has no bearing on the asymptotic dependence upon $k$. 


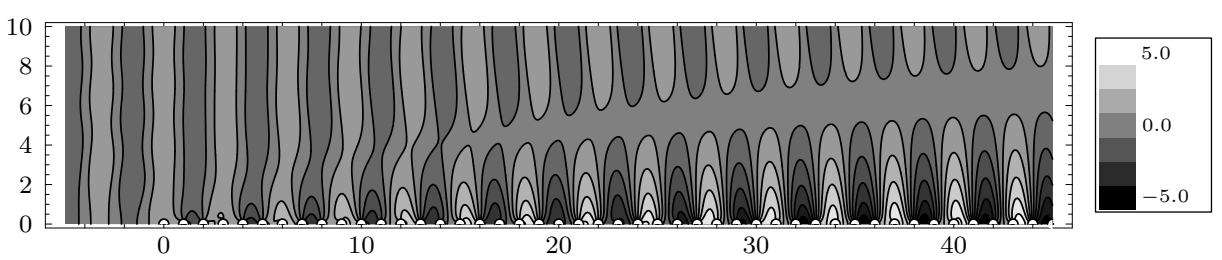

FIG. 5.3. Contour plot of the real part of the total field, $\operatorname{Re}[\phi]$ at head-on incidence with $a=0.25$ and $k=2.0$.

The approximation (5.11) is nonuniform in the sense that it is singular at the shadow boundaries, where $\psi_{m}=\theta$. The uniform counterpart to (5.11) is given by

$$
\begin{aligned}
& \phi_{\text {sc }} \sim \mathrm{e}^{\mathrm{i} k r}\left\{\mathrm{e}^{-\mathrm{i} \pi / 4}\right. \sqrt{\frac{2}{\pi k r}} h(\theta)-w\left(\mathrm{e}^{\mathrm{i} \pi / 4} \zeta_{0}\right)+\frac{\mathrm{e}^{\mathrm{i} \pi / 4}}{\zeta_{0} \sqrt{\pi}}-\sum_{m \in \mathcal{M}} \frac{\mathcal{G}\left(\psi_{m}\right) \mathrm{e}^{\mathrm{i} \pi / 4}}{\sqrt{\pi} \sqrt[4]{k r}} \sqrt{\sec \frac{\psi_{m}-\theta}{2}} \\
&\left.\times\left[\mathrm{e}^{\mathrm{i}\left(3 \pi / 8-\zeta_{m}^{2} / 2\right)} D_{-1 / 2}\left(\sqrt{2} \mathrm{e}^{-\mathrm{i} \pi / 4} \zeta_{m}\right)-\frac{1}{\sqrt[4]{2}\left(-\zeta_{m}\right)^{1 / 2}}\right]\right\}, \quad(5.14)
\end{aligned}
$$

in which $\zeta_{m}=\sqrt{2 k r} \sin \left(\frac{1}{2}\left(\theta-\psi_{m}\right)\right)$. Here, the term involving $\zeta_{0}$ removes the singularity at $\theta=0$. Note that the resonant mode actually has no region of existence, since $\zeta_{0} \geq 0$. Nevertheless, its influence can be felt as $\theta \rightarrow 0$, since for small $z$, $w(z)=\mathrm{e}^{-z^{2}}[1+O(z)]$. As in the nonresonant case, in the limit $\theta \rightarrow 0$, (5.14) accurately represents a contribution to the far field, though this is not necessarily the most significant. The final term involves the parabolic cylinder function $D_{-1 / 2}(\cdot)$, and can be obtained using methods outlined in [30], although this is by no means an easy procedure. However, it is relatively straightforward to check that (5.14) is correct. Firstly, it is continuous across all of the shadow boundaries. To see this, we use (5.10) and (5.12) to show that the singular behaviour of the term involving $h(\theta)$ at $\theta=\psi_{m}$ is cancelled by the series. Also, for large $|\zeta|$, we have from [31, §9.246]

$$
\mathrm{e}^{\mathrm{i}\left(3 \pi / 8-\zeta^{2} / 2\right)} D_{-1 / 2}\left(\sqrt{2} \mathrm{e}^{-\mathrm{i} \pi / 4} \zeta\right)=\frac{1+\mathrm{i} \sqrt{2} \mathrm{H}(-\zeta) \mathrm{e}^{-\mathrm{i} \zeta^{2}}}{\sqrt[4]{2}(-\zeta)^{1 / 2}}+O\left(\zeta^{-5 / 2}\right),
$$

where $\mathrm{H}(\cdot)$ is the Heaviside unit function, and $\left(-\zeta_{m}\right)^{1 / 2}$ is either positive real or negative imaginary. If we now use this expansion, along with (4.9), in (5.14), we retrieve (5.11), as we should expect. Note that $D_{-1 / 2}(0) \approx 1.216$, therefore in the vicinity of the shadow boundary, the scattered field is $O\left((k r)^{-1 / 4}\right)$, as in the case of point scatterers [29].

Figure 5.3 shows a contour plot of the real part of the total field, at head-on incidence with $a=0.25$ and $k=2.0$. The cancellation of the incident field close to the array is clearly apparent, so that the symmetric Rayleigh-Bloch wave (for which $|\alpha| \approx 0.542)$ is clearly visible.

The branch point contributions that are significant at higher frequencies can most easily be observed in a plot of the scattered field. Thus, Figure 5.4 shows a contour plot of $\operatorname{Re}\left[\phi_{\mathrm{sc}}\right]$, with $a=0.25$, and $k=8.0$, using Dirichlet boundary conditions. Values of $\psi_{m}$ for which $m \in \mathcal{M}$ and the associated values of $\left|\mathcal{G}\left(\psi_{m}\right)\right|$ are shown in Table 5.2. Far field plots with $r=8$ are shown in Figure 5.5, with shadow boundaries indicated by black disks. The dashed line is computed from equation (5.14), whereas for the solid line, $D_{-1 / 2}\left(\sqrt{2} \mathrm{e}^{-\mathrm{i} \pi / 4} \zeta\right)$ is replaced by $-\mathrm{i} D_{-1 / 2}\left(\sqrt{2} \mathrm{e}^{-\mathrm{i} \pi / 4}|\zeta|\right)$ for $\zeta<0$ so 


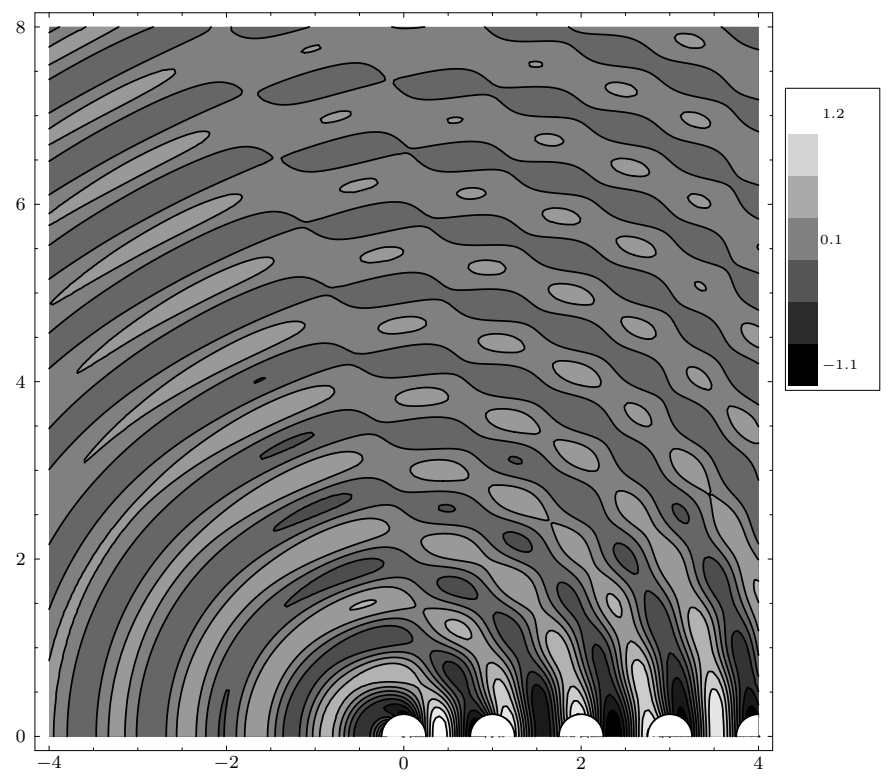

Fig. 5.4. Contour plot of $\operatorname{Re}\left[\phi_{\mathrm{sc}}\right]$, at head-on incidence with $a=0.25 k=8.0$ using Dirichlet boundary conditions.

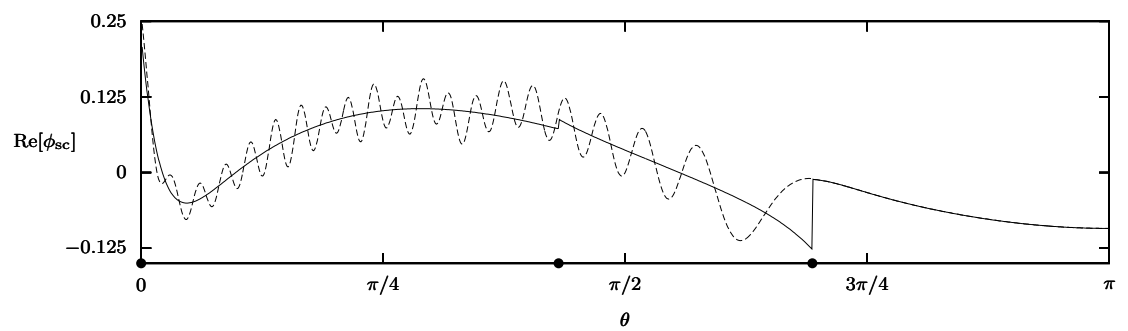

FIG. 5.5. Far field plots for the parameters used in Figure 5.4, with $r=8$. Correction terms are included for both shadow boundaries; the dashed line includes branch point contributions whereas the solid line does not.

as to deactivate the branch point contributions. This plot is therefore discontinuous at the shadow boundaries, and the size of the discontinuities are consistent with the values of $\left|\mathcal{G}\left(\psi_{m}\right)\right|$ in Table 5.2. There are three regions to consider. For $\theta \gtrsim 0.69 \pi$, no branch point contributions are present in the field, and the circular wave dominates. For smaller observation angles, a branch point contribution is activated, causing interference. Notice in particular the strong field close to the shadow boundary, where (5.14) predicts $O\left((k r)^{-1 / 4}\right)$ behaviour. A second branch point contribution is active when $\theta \lesssim 0.43 \pi$. This is somewhat weaker and has a more limited effect on the field pattern. A final possibility is that of double resonance, which requires that $k=n \pi$, $n \in \mathbb{N}$. In this case, if $\cos \psi_{m}=1$, then $\cos \psi_{m-n}=-1$, so that modes $m$ and $m-n$ are inwards and outwards resonant, respectively. Once the coefficients $B_{n}$ for the infinite array problem have been obtained using the method in [17], the computation of the coefficients $C_{m}^{p}$ for this case presents no special difficulty beyond those already discussed. However, the determination of the far field pattern involves a significant additional complication, and will therefore appear in a future paper. 
TABLE 5.2

Shadow boundary locations and values of $\mathcal{G}\left(\psi_{m}\right)$ for the plot shown in Figure 5.1.

\begin{tabular}{c|cc}
$m$ & $\psi_{m}$ & $\left|\mathcal{G}\left(\psi_{m}\right)\right|$ \\
\hline-2 & $0.69 \pi$ & 0.39 \\
-1 & $0.43 \pi$ & 0.12
\end{tabular}

6. Conclusion. Problems involving semi-infinite arrays are notoriously difficult to solve accurately because the inevitable spatial truncation that has to be made can introduce significant errors. We have shown how infinite array subtraction, together with a novel filtering approach, can be used to obtain accurate solutions which can be computed efficiently for two-dimensional acoustic scattering by a semi-infinite array of rigid or soft circles. Unlike the case of isotropic point scatterers solved previously by one of the authors [8] this case is made considerably more complicated by the presence of Rayleigh-Bloch surface waves which can be excited along the array. We have presented methods which enable the amplitude of these modes to be computed accurately for the first time.

In nonresonant and outward resonant cases, the far field away from the array has been shown to be composed of the sum of a finite number of plane waves propagating in different directions and a circular wave emanating from the edge of the array. At inward resonance, the field can include additional terms, that are neither circular waves nor plane waves. Uniform asymptotic expansions that vary continuously across all shadow boundaries have been derived.

One of the main reasons for studying semi-infinite arrays is that they provide a tool with which to analyse large finite arrays. Thus we intend to use the techniques presented in this paper to study scattering by a long finite array under the assumption that the ends of the array are far enough apart to be treated separately.

Appendix. If we define

$$
\Delta_{m}^{p}=\Gamma_{m}^{p}-\mathrm{e}^{\mathrm{i} \widetilde{\beta}} \Gamma_{m}^{p-1}, \quad p=2,3, \ldots,
$$

then from (3.16) and (3.19) we obtain

$$
E_{m}^{p}+\sum_{n=-\infty}^{\infty} Z_{n} \sum_{\substack{j=0 \\ \neq p}}^{\infty} E_{n}^{j} X_{n-m}^{j p} \mathrm{H}_{n-m}(k|j-p|)=\Delta_{m}^{p}-\mathrm{e}^{\mathrm{i} k} \Delta_{m}^{p-1},
$$

$p=2,3, \ldots, m \in \mathbb{Z}$. The equations in which $p=0$ and $p=1$ now require special treatment. From (3.19), we have

$$
D_{m}^{p}=\sum_{j=0}^{p} \mathrm{e}^{\mathrm{i}(p-j) k} E_{m}^{j}, \quad p=0,1,2, \ldots
$$

Setting $p=1$ in (3.16) we find that

$$
\begin{aligned}
\mathrm{e}^{\mathrm{i} k} E_{m}^{0} & +E_{m}^{1}+\sum_{n=-\infty}^{\infty} E_{n}^{0} Z_{n} \mathrm{H}_{n-m}(k) \\
& +\sum_{n=-\infty}^{\infty}(-1)^{n-m} Z_{n} \sum_{j=0}^{\infty} E_{n}^{j} \mathrm{e}^{\mathrm{i}(1-j) k} \sum_{l=\max (j-1,1)}^{\infty} \mathrm{e}^{\mathrm{i} k l} \mathrm{H}_{n-m}(k l)=\Delta_{m}^{1}
\end{aligned}
$$


Finally from (3.17), for $p=0$,

$$
E_{m}^{0}+\sum_{n=-\infty}^{\infty} Z_{n}(-1)^{n-m} T_{n m}=\Gamma_{m}^{0}
$$

where

$$
T_{n m}=\sum_{j=0}^{\infty} \sum_{q=0}^{j} E_{n}^{q} \mathrm{e}^{\mathrm{i}(j-q) k} \sum_{l=\max (j, 1)}^{\infty} \mathrm{e}^{\mathrm{i} \tilde{\beta}(l-j)} \mathrm{H}_{n-m}(k l) .
$$

This expression can be rearranged so that the sum over $j$ becomes innermost. After evaluating the finite geometric series that appears, we obtain

$$
T_{n m}=\frac{1}{\mathrm{e}^{\mathrm{i} \tilde{\beta}}-\mathrm{e}^{\mathrm{i} k}} \sum_{q=0}^{\infty} E_{n}^{q} \sum_{l=\max (q, 1)}^{\infty}\left(\mathrm{e}^{\mathrm{i} \widetilde{\beta}(1+l-q)}-\mathrm{e}^{\mathrm{i} k(1+l-q)}\right) \mathrm{H}_{n-m}(k l) .
$$

The sum over $l$ is again easily expressed in terms of the sums $S_{n}^{p}$ defined in (3.4).

To reconstruct the coefficients $C_{m}^{p}$, we substitute (A.3) into (3.14), reverse the order of the summations and evaluate the resulting geometric series to obtain

$$
\begin{aligned}
C_{m}^{p} & =\frac{\mathrm{e}^{\mathrm{i} p \tilde{\beta}}}{1-\mathrm{e}^{\mathrm{i}(k-\tilde{\beta})}} \sum_{q=0}^{p} E_{m}^{q} \mathrm{e}^{-\mathrm{i} q \tilde{\beta}}+\frac{\mathrm{e}^{\mathrm{i} p k}}{1-\mathrm{e}^{-\mathrm{i}(k-\tilde{\beta})}} \sum_{q=0}^{p} E_{m}^{q} \mathrm{e}^{-\mathrm{i} q k} \\
& =\frac{\mathrm{e}^{\mathrm{i} p \tilde{\beta}}}{1-\mathrm{e}^{\mathrm{i}(k-\tilde{\beta})}} \sum_{q=0}^{p} E_{m}^{q} \mathrm{e}^{-\mathrm{i} q \tilde{\beta}}+\frac{D_{m}^{p}}{1-\mathrm{e}^{-\mathrm{i}(k-\tilde{\beta})}}
\end{aligned}
$$

where we have used (3.19) to evaluate the second (telescopic) series.

The Rayleigh-Bloch amplitude $\alpha$ can then be retrieved by letting $p \rightarrow \infty$ and using (3.18):

$$
\alpha \widetilde{B}_{m}=\frac{1}{1-\mathrm{e}^{\mathrm{i}(k-\tilde{\beta})}} \sum_{q=0}^{\infty} E_{m}^{q} \mathrm{e}^{-\mathrm{i} q \tilde{\beta}} .
$$

Once again, asymptotic acceleration can be used in the approximate evaluation of this series, once spatial truncation has been applied.

Acknowledgement. IT is supported by EPSRC under grant EP/C510941/1.

\section{REFERENCES}

[1] Boris Tomasic and Alexander Hessel. Analysis of finite arrays-a new approach. IEEE Trans. Antennas Propagat., 47:555-565, 1999.

[2] D. S. Janning and B. A. Munk. Effects of surface waves on the currents of truncated periodic arrays. IEEE Trans. Antennas Propagat., 50(9):1254-1265, 2002.

[3] R. W. Ziolkowski and N. Engheta. Metamaterials special issue introduction. IEEE Trans. Antennas Propagat., 51(10):2546-2549, 2003.

[4] M. Kashiwagi. Hydrodynamic interactions among a great number of columns supporting a very large flexible structure. J. Fluids and Structures, 14:1013-1034, 2000.

[5] H. Kagemoto, M. Murai, M. Saito, B. Molin, and Š. Malenica. Experimental and theoretical analysis of the wave decay along a long array of vertical cylinders. J. Fluid Mech., 456:113$135,2002$. 
[6] Norman L. Hills and Samuel N. Karp. Semi-infinite diffraction gratings-I. Comm. Pure Appl. Maths, 18:203-233, 1965.

[7] R. F. Millar. Plane wave spectra in grating theory. III. Scattering by a semiinfinite grating of identical cylinders. Can. J. Phys., 42:1149-1184, 1964.

[8] C. M. Linton and P. A. Martin. Semi-infinite arrays of isotropic point scatterers. A unified approach. SIAM J. Appl. Math., 64(3):1035-1056, 2004.

[9] R. Porter and D. V. Evans. Rayleigh-Bloch surface waves along periodic gratings and their connection with trapped modes in waveguides. J. Fluid Mech., 386:233-258, 1999.

[10] C. M. Linton and M. McIver. The existence of Rayleigh-Bloch surface waves. J. Fluid Mech., 470:85-90, 2002.

[11] A. N. Norris and Z. Wang. Bending-wave diffraction from strips and cracks on thin plates. Q. J. Mech. Appl. Math., 47:607-627, 1994.

[12] H. D. Maniar and J. N. Newman. Wave diffraction by a long array of cylinders. J. Fluid Mech., 339:309-330, 1997.

[13] C. M. Linton and D. V. Evans. The interaction of waves with arrays of vertical circular cylinders. J. Fluid Mech., 215:549-569, 1990.

[14] V. Twersky. Elementary function representation of Schlömilch series. Arch. Rational Mech. Anal., 8:323-332, 1961.

[15] C. M. Linton. The Green's function for the two-dimensional Helmholtz equation in periodic domains. J. Engng. Math., 33:377-402, 1998.

[16] P. A. Martin. Multiple Scattering. Interaction of Time-Harmonic Waves with $N$ Obstacles. Cambridge University Press, 2006.

[17] C. M. Linton and I. Thompson. Resonant effects in scattering by periodic arrays. To appear in Wave Motion.

[18] P. McIver, C. M. Linton, and M. McIver. Construction of trapped modes for wave guides and diffraction gratings. Proc. Roy. Soc. Lond., A, 454:2593-2616, 1998.

[19] D. V. Evans and R. Porter. Trapping and near-trapping by arrays of cylinders in waves. J. Engng. Math., 35:149-179, 1999.

[20] Anne-Sophie Bonnet-Bendhia and Felipe Starling. Guided waves by electromagnetic gratings and non-uniqueness examples for the diffraction problem. Math. Meth. in the Appl. Sci., 17:305-338, 1994.

[21] M. Nishimoto and H. Ikuno. Space-wavenumber analysis of field scattered from a semi-infinite strip grating. Electrical Engineering in Japan, 132:1-8, 2000.

[22] R. Porter and D. V. Evans. Scattering of flexural waves by multiple narrow cracks in ice sheets floating on water. Wave Motion, 43:425-443, 2005.

[23] C. M. Linton. Schlömilch series that arise in diffraction theory and their efficient computation. J. Phys. A, 39:3325-3339, 2006.

[24] R. J. Mailloux. Excitation of a surface wave along an infinite Yagi-Uda array. IEEE Trans. Antennas Propagat., 13:719-724, 1965.

[25] J. Paul Skinner and Peter J. Collins. A one-sided version of the Poisson sum formula for semi-infinite array Green's functions. IEEE Trans. Antennas Propagat., 45(4):601-607, 1997.

[26] M. A. Sumbatyan and A. Scalia. Equations of Mathematical Diffraction Theory. Chapman \& Hall/CRC, 2005.

[27] Ian Thompson. An improved uniform approximation for diffraction integrals. Proc. Roy. Soc. Lond., A, 462:1341-1353, 2006

[28] M. Abramowitz and I. A. Stegun. Handbook of Mathematical Functions. Dover Publications, New York, 1965.

[29] Norman L. Hills. Semi-infinite diffraction gratings. II. Inward resonance. Comm. Pure Appl. Maths, 18:389-395, 1965

[30] Norman Bleistein and Richard A. Handelsman. Asymptotic Expansions of Integrals. Dover Publications, New York, 1986. Originally published in 1975.

[31] I. S. Gradshteyn and I. M. Ryzhik. Tables of Integrals, Series and Products. Academic Press, New York, 6th edition, 2000. 